\title{
STABILITY AND DISSIPATIVITY ANALYSIS FOR NEUTRAL TYPE STOCHASTIC MARKOVIAN JUMP STATIC NEURAL NETWORKS WITH TIME DELAYS
}

\author{
Yang $\mathrm{Cao}^{1}$, R. Samidurai ${ }^{2}$, and R. Sriraman ${ }^{2}$ \\ ${ }^{1}$ Department of Mechanical Engineering, \\ The University of Hong Kong, Pokfulam, Hong Kong \\ ${ }^{2}$ Department of Mathematics, Thiruvalluvar University, \\ Vellore 631 115, India \\ E-mail: caoyeacy@hku.hk,sriraman225@gmail.com,samidurair@gmail.com
}

Submitted:8th September 2018; Accepted: 21th November 2018

\begin{abstract}
This paper studies the global asymptotic stability and dissipativity problem for a class of neutral type stochastic Markovian Jump Static Neural Networks (NTSMJSNNs) with time-varying delays. By constructing an appropriate Lyapunov-Krasovskii Functional (LKF) with some augmented delay-dependent terms and by using integral inequalities to bound the derivative of the integral terms, some new sufficient conditions have been obtained, which ensure that the global asymptotic stability in the mean square. The results obtained in this paper are expressed in terms of Strict Linear Matrix Inequalities (LMIs), whose feasible solutions can be verified by effective MATLAB LMI control toolbox. Finally, examples and simulations are given to show the validity and advantages of the proposed results.
\end{abstract}

Keywords: Static neural networks, Dissipativity analysis, Markovian jump, Time-varying delays.

\section{Introduction}

Over the past twenty years, stability analysis of Neural Networks (NNs) have been extensively investigated for their widespread applications in many areas such as imaging processing, engineering optimization, pattern recognition, associative memories, computer version and so on [1-4]. Such an application has strongly dependent on the stability of the stable equilibrium point of NNs, and such an equilibrium point may affect by the existence of time delays and noise disturbance [5-8]. Consequently, the investigation of the stability of NNs with these two aspects is very imperative. There- fore, a great deal of research consideration is dedicated to studying the stability of NNs with time delays, numerous noteworthy results related to this problem have been obtained recently, see $[1-5,8$ 13, 16-34].

As we know, Markovian jumping systems (MJSs) are viewed as a special class of hybrid systems, which provide an efficient way to represent a class of dynamic systems subject to random changes in their structures and found their applications in fault-tolerant systems, manufactory processes $[14,15]$. Therefore, the stability of NNs with Markovian jump parameters have received much research consideration, many significant results re- 
lated to this problem have been well documented, see $[5,8-10,12-13,16,23,40]$. For example, in [5], LMI-based sufficient conditions have been obtained for Markovian jump NNs including the relative information on the mode-dependent additive time-varying delays. In [10], the exponential stability criteria for a class of stochastic NNs with both mixed time delays and Markovian jump parameters has been investigated, novel Lyapunov functional has been considered into the framework. By employing stochastic analysis, the problem of stability of stochastic Markovian switching static NNs with asynchronous mode-dependent delays has been investigated in the work [12]. On the other hand, as pointed out in [17] that the NN could be stabilized or destabilized by certain stochastic inputs, so for, it is of essential significance to consider stochastic disturbance on the stability analysis of NNs. Recently, there are several results regarding the stability of NNs with stochastic effects have been published, see [18-26]. For example, in [19], a novel LKF is constructed, which includes the relative information of the derivative of the past state, some LMI-based stability criteria are derived for the uncertain stochastic nonlinear systems. By constructing an augmented LKF, a class of uncertain stochastic NNs with both neutral type and interval timevarying delays has been investigated in the reference [20]. Meanwhile, many application systems can be structured as a differential form of neutral type, which will contain the information about the derivative of the past state. Such phenomenon constantly encountered in the field of heat exchangers, chemical reactors, population dynamics systems [28]. Due to the fact that the neutral type NNs have received much research attention, many interesting results on this topic have been reported recently [29-34].

Nowadays, there has been a rapidly growing research attention has been paid to the issue of dissipativity of NNs, which has strong application background in engineering fields such as networks, circuit systems, and control theory [35, 36]. In [35], dissipative systems were initially developed, after that the concept further extended in [36]. Besides, the dissipativity theory deals with a new computational approach through the appropriate parameters $(Q, S, R)$ compared with other methods. Thus, the study of dissipativity analysis has received much research consideration from the researchers, many significant results have been published, see [4], [3741]. To the best of authors knowledge, the problem of stability and dissipativity analysis and the design of stochastic disturbance for neutral-type Markovian jump static NNs have not been completely investigated, it remains essential and challenging. This circumstance motivates our present study.

Inspired by the above discussions, in this paper, our research efforts are mainly focused to investigate the global asymptotic stability and dissipativity analysis for a class of NTSMJSNNs with timevarying delays. The contributions of this paper are listed as follows: (i) By constructing an appropriate LKF with some augmented delay-dependent terms, by employing integral inequalities, some new LMIbased sufficient conditions have been established, which ensure that the global asymptotically stability in the mean square. (ii) The results obtained in this paper are further extended to the dissipativity analysis with nonlinear stochastic disturbance. (iii) The calculated maximum allowable upper bounds (MAUBs) $\eta$ based on various methods are compared in the illustrative section, which is guaranteed less conservative than recently existing works. (iv) Finally, three illustrative examples and simulations are presented to show the effectiveness of the proposed results.

The problem is formally defined in the next Section. The stability and dissipativity criteria are presented in Section 3 and 4, respectively. In Section 5 , numerical examples are given. At last, the conclusion is drawn in Section 6.

Notations: Throughout this paper, $\mathbb{R}^{n}$ and $\mathbb{R}^{m \times n}$ denotes the $n$-dimensional Euclidean space and the set of $m \times n$ real matrices, respectively. The superscript $\mathbf{X}^{T}$ represents the transpose of $\mathbf{X} . \mathbf{P}>$ 0 means that $\mathbf{P}$ is the symmetric positive definite matrix. denotes the elements below the main diagonal of a symmetric block matrix. $(\Omega, \mathscr{F}, \mathscr{P})$ is complete probability space with a natural filtration $\left\{\mathscr{F}_{t}\right\}_{t \geq 0}$ satisfying the usual conditions. $\mathbf{I}_{n}$ represents the identity matrix with appropriate dimensions. $\operatorname{diag}\{$.$\} denote the block diagonal matrix.$ $\mathcal{L}_{2}[0, \infty)$ is the space of an $n$-dimensional square integral vector function on $[0, \infty) . \mathbb{E}\{\cdot\}$ denotes the mathematical expectation operator with respect to $\mathscr{P}$. 


\section{Problem formulation and prelim- inaries}

Let $\{\rho(t), t \geq 0\}$ is a right-continuous Markov chain defined on $(\Omega, \mathscr{F}, \mathscr{P})$ and take discrete values in a finite state space $S=\{1,2, \ldots, N\}$ with transition probability matrix $\Pi=\left[\pi_{i j}\right]_{N \times N}$ given by

$$
\begin{aligned}
& \operatorname{Pr}\{\rho(t+\triangle t=j \mid \rho(t)=i\} \\
& = \begin{cases}\pi_{i j} \triangle t+o(\triangle t), & \text { if } i \neq j, \\
1+\pi_{i i} \triangle t+o(\triangle t), & \text { if } i=j,\end{cases}
\end{aligned}
$$

where $\Delta t>0$ and $\lim _{\triangle t \rightarrow 0^{+}} \frac{o(\Delta t)}{\Delta t}=0, \pi_{i j} \geq 0$ is the transition rate from $i$ to $j$, If $i \neq j$ while $\pi_{i i}=$ $-\sum_{j=1}^{N} \pi_{i j}$.

Now, we consider the following neutral type Markovian jump static NNs with time varying delays

$$
\left\{\begin{aligned}
\dot{q}(t)= & -\mathbf{D}(\rho(t)) q(t)+f(\mathbf{W}(\rho(t)) q(t \\
& -\eta(t)))+\mathbf{A}(\rho(t)) \dot{q}(t-h(t)), \\
q(t)= & \psi(t), t \in[-\rho, 0],
\end{aligned}\right.
$$

For the purpose of simplicity, let $\rho(t)=i$. Then $\mathbf{D}(\rho(t))=\mathbf{D}_{i}, \mathbf{W}(\rho(t))=\mathbf{W}_{i}$ and $\mathbf{A}(\rho(t))=\mathbf{A}_{i}$. Now the system (3) can be written as

$$
\left\{\begin{aligned}
\dot{q}(t)= & -\mathbf{D}_{i} q(t) \\
& +f\left(\mathbf{W}_{i} q(t-\eta(t))\right)+\mathbf{A}_{i} \dot{q}(t-h(t)), \\
q(t)= & \psi(t), t \in[-\rho, 0]
\end{aligned}\right.
$$

where $q(t)=\left[q_{1}(t), q_{2}(t), \ldots, q_{n}(t)\right]^{T} \in \mathbb{R}^{n}$ is the state vector. The diagonal matrix $\mathbf{D}_{i}=\mathbf{D}(\rho(t))=$ $\operatorname{diag}\left\{d_{1}(\rho(t)), d_{2}(\rho(t)), \ldots, d_{n}(\rho(t))\right\}$ has positive entries $d_{i}(\rho(t))>0(i=1,2, \ldots, n)$. The matrices $\mathbf{W}_{i}=\mathbf{W}(\rho(t))$ and $\mathbf{A}_{i}=\mathbf{A}(\rho(t))$ represent the connection weight matrices. $\psi(t)$ is a vectorvalued initial function defined on $[-\rho, 0]$, where $\rho=\max \{\eta, h\}$.

A1: The delays $\eta(t)$ and $h(t)$ are time-varying continuous functions that satisfy

$$
\left\{\begin{array}{l}
0 \leq \eta(t) \leq \eta, \quad \dot{\eta}(t) \leq \mu, \\
0 \leq h(t) \leq h, \quad \dot{h}(t) \leq h_{d}
\end{array}\right.
$$

where $\eta, h, \mu$ and $h_{d}$ are some constants.

A2: The neuron activation function $f_{j}(\cdot) j=$ $1,2, \ldots, n$ in (4) is continuous, bounded and satisfies,

$$
\gamma_{j}^{-} \leq \frac{f_{j}(u)-f_{j}(v)}{(u-v)} \leq \gamma_{j}^{+}
$$

$f_{j}(0)=0, u, v \in \mathbb{R}, u \neq v$ and $\gamma_{j}^{-}$and $\gamma_{j}^{+}$are known real constants.

which is equivalent to

$$
\begin{aligned}
& {\left[f_{j}(u)-f_{j}(v)-\gamma_{j}^{-}(u-v)\right]\left[f_{j}(u)-f_{j}(v)\right.} \\
& \left.-\gamma_{j}^{+}(u-v)\right] \leq 0, j=1,2, \ldots, n .
\end{aligned}
$$

If $v=0$ in (6), we have

$$
\gamma_{j}^{-} \leq \frac{f_{j}(u)}{u} \leq \gamma_{j}^{+}, j=1,2, \ldots, n,
$$

which is equivalent to $\left[f_{j}(u)-\gamma_{j}^{-} u\right]\left[f_{j}(u)-\gamma_{j}^{+} u\right] \leq$ $0, j=1,2, \ldots, n$.

Now, we consider the following NTSMJSNNs with time-varying delays

$$
\left\{\begin{aligned}
d q(t)= & {\left[-\mathbf{D}_{i} q(t)+f\left(\mathbf{W}_{i} q(t-\eta(t))\right)\right.} \\
& \left.+\mathbf{A}_{i} \dot{q}(t-h(t))+u(t)\right] d t \\
& +\sigma(t, q(t), q(t-\eta(t)), i) d \omega(t) \\
p(t)= & f\left(\mathbf{W}_{i} q(t)\right) \\
q(t)= & \psi(t), t \in[-\rho, 0]
\end{aligned}\right.
$$

where $p(t)$ is the output of the NNs (7), $u(t)$ is the external disturbance input which belongs to $\mathcal{L}_{2}[0, \infty) . \quad \sigma(t, q(t), q(t-\eta(t)), i)$ is the linear or nonlinear stochastic perturbation. $\omega(t)=$ $\left[\omega_{1}(t), \ldots, \omega_{n}(t)\right]^{T} \in \mathbb{R}^{m}$ is an $m$-dimensional Brownian motion defined on $(\Omega, \mathscr{F}, \mathscr{P})$ that satisfies usual conditions.

A3: The stochastic perturbation $\sigma(t, q(t), q(t-$ $\eta(t)), i)$ is locally Lispchitz continuous and satisfies the linear growth condition

$$
\begin{aligned}
& \operatorname{tr}\left\{\boldsymbol{\sigma}^{T}(t, q(t), q(t-\eta(t)), i) \sigma(t, q(t), q(t-\right. \\
& \eta(t)), i)\} \leq\left\|\mathbf{F}_{1 i} q(t)\right\|^{2}+\left\|\mathbf{F}_{2 i} q(t-\eta(t))\right\|^{2},
\end{aligned}
$$

where $\operatorname{tr}(\cdot)$ stands for $\operatorname{tr}, \mathbf{F}_{1 i}$ and $\mathbf{F}_{1 i}(i \in S)$ are real matrices with suitable dimensions. Supposed the term $q^{T}(t) P_{i} q(t)$ is taken into the account of LKF $\mathbb{V}(t, q(t), i)$, by Ito's differential rule, there exists a nonlinear stochastic term $\operatorname{tr}\left\{\sigma^{T}(t, q(t), q(t-\right.$ $\left.\eta(t)), i) \mathbf{P}_{i} \sigma(t, q(t), q(t-\eta(t)), i)\right\}$. In order to obtain the less conservative stability criteria for NN (7), the following restrictive condition is required

$$
\mathbf{P}_{i} \leq \lambda_{i} \mathbf{I}
$$

From (9) and (10), we have

$$
\begin{aligned}
& \operatorname{tr}\left\{\boldsymbol{\sigma}^{T}(t, q(t), q(t-\eta(t)), i) \mathbf{P}_{i} \sigma(t, q(t), q(t\right. \\
& -\eta(t)), i)\} \leq \lambda_{i}\left\|\mathbf{F}_{1 i} q(t)\right\|^{2} \\
& +\lambda_{i}\left\|\mathbf{F}_{2 i} q(t-\eta(t))\right\|^{2}
\end{aligned}
$$


similar to [11, 12], to reduce the conservatism further, the following restrictive condition (10) can be relaxed according to the next two assumptions made on the form of the $(\sigma(t, q(t), q(t-\eta(t)), i)$.

A4: $\sigma(t, q(t), q(t-\eta(t)), i)$ is nonlinear and can be written as

$$
\begin{aligned}
& \sigma(t, q(t), q(t-\eta(t)), i)=\mathbf{H}_{1 i}(t) q(t) \\
& +\mathbf{H}_{2 i}(t) q(t-\eta(t)),
\end{aligned}
$$

where $\mathbf{H}_{1 i}(t)$ and $\mathbf{H}_{1 i}(t)$ are nonlinear diagonal matrix functions.

A5: The matrices $\mathbf{F}_{1 i}$ and $\mathbf{F}_{1 i}$ in (9) are diagonal matrices

$$
\begin{aligned}
& \mathbf{F}_{1 i}=\operatorname{diag}\left\{u_{i 1}, u_{i 2}, \ldots, u_{i n}\right\}, \\
& \mathbf{F}_{1 i}=\operatorname{diag}\left\{v_{i 1}, v_{i 2}, \ldots, v_{i n}\right\},
\end{aligned}
$$

where $u_{i j}>0, v_{i j}>0, i, j=1,2, \ldots, n$ are some scalars.

From A4 and A5, the condition (10) can be relaxed as

$$
\mathbf{P}_{i} \leq \Lambda_{i}=\operatorname{diag}\left\{\lambda_{i 1}, \lambda_{i 2}, \ldots, \lambda_{i n}\right\}
$$

where $\Lambda_{i j}>0, j=1,2, \ldots, n$. As a result of (18) is less restrictive and conservative than (10). The detailed proof can be found in $[11,12$.

Suppose a 2D stochastic perturbation $\sigma(t, q(t), q(t-\eta(t)), i)$ can be given by

$$
\begin{aligned}
& \sigma(t, q(t), q(t-\eta(t)), i)= \\
& \sqrt{0.080}\left[\begin{array}{l}
\sin t q_{1}(t)+\cos ^{2} t q_{1}(t-\eta(t)) \\
\cos t q_{2}(t)+\sin ^{2} t q_{2}(t-\eta(t))
\end{array}\right]
\end{aligned}
$$

which can be described by (15) with

$$
\begin{aligned}
& \mathbf{H}_{1 i}=\sqrt{0.080}\left[\begin{array}{cc}
\sin t & 0 \\
0 & \cos t
\end{array}\right], \\
& \mathbf{H}_{2 i}=\sqrt{0.080}\left[\begin{array}{cc}
\cos ^{2} t & 0 \\
0 & \sin ^{2} t
\end{array}\right]
\end{aligned}
$$

Hence, we have

$$
\begin{aligned}
& \operatorname{tr}\left\{\sigma^{T}(t, q(t), q(t-\eta(t)), i) \sigma(t, q(t), q(t\right. \\
& -\eta(t)), i)\}=\sigma^{T}(t, q(t), q(t-\eta(t)), i) \sigma(t, \\
& q(t), q(t-\eta(t)), i) \\
& \leq 2\left\|\sqrt{0.080}\left[\begin{array}{cc}
\sin t & 0 \\
0 & \cos t
\end{array}\right] q(t)\right\|^{2} \\
& +2\left\|\sqrt{0.080}\left[\begin{array}{cc}
\cos ^{2} t & 0 \\
0 & \sin ^{2} t
\end{array}\right] q(t-\eta(t))\right\|^{2} \\
& \leq\left\|\left[\begin{array}{cc}
\sqrt{0.16} & 0 \\
0 & \sqrt{0.16}
\end{array}\right] q(t)\right\|^{2} \\
& +\left\|\left[\begin{array}{cc}
\sqrt{0.16} & 0 \\
0 & \sqrt{0.16}
\end{array}\right] q(t-\eta(t))\right\|^{2} .
\end{aligned}
$$

which implies that $\mathbf{F}_{1 i}$ and $\mathbf{F}_{2 i}$ in (13) are satisfied with

$$
\mathbf{F}_{1 i}=\mathbf{F}_{2 i}=\sqrt{0.16}=0.4 \mathbf{I}
$$

The following definition and lemmas, are needed to obtain the main results.

Definition 2.1. The NTSMJSNNs (7) is said to be mean-square stable, if for any $\varepsilon>0$ there exists a scalar $\vartheta(\varepsilon)>0$ such that $\mathbb{E}\left\{\|q(t)\|^{2}\right\} \leq \varepsilon, t>0$, whenever $\sup \mathbb{E}\left\{\|\psi(t)\|^{2}\right\} \leq \vartheta(\varepsilon)$. In addition, $-\rho \leq t \leq 0$

if $\lim _{t \rightarrow \infty} \mathbb{E}\left\{\|q(t)\|^{2}\right\}=0$ for any initial conditions, then the NTSMJSNNs (7) is said to be mean-square asymptotically stable.

Definition 2.2. The NTSMJSNNs (7) is strictly $(Q, S, R)-\gamma-$ dissipative for any $t_{r} \geq 0$ and scalar $\gamma>0$, if under zero initial state, the following condition is fulfilled:

$$
\mathbb{E}\left\{\mathcal{G}\left(u, p, t_{r}\right)\right\} \geq \mathbb{E}\left\{\gamma\langle u, u\rangle_{t_{r}}\right\} .
$$

Remark 2.3. As per the definition (2.2), the energy supply function $\mathcal{G}$ related to the system (7) defined by

$$
\begin{aligned}
& \mathcal{G}\left(u, p, t_{r}\right)=\langle p, Q p\rangle_{t_{r}}+2\langle p, \mathcal{S} u\rangle_{t_{r}} \\
& +\langle u, \mathcal{R} u\rangle_{t_{r}}, \forall t_{r} \geq 0,
\end{aligned}
$$

where $Q, \mathcal{S}, \mathcal{R} \in \mathbb{R}^{m \times n}$ with $Q, \mathcal{R}$ are symmetric. The notations $\langle p, Q p\rangle_{t_{r}},\langle p, \mathcal{S} u\rangle_{t_{r}}$ and $\langle u, \mathcal{R} u\rangle_{t_{r}}$ are represents $\int_{0}^{t_{r}} p^{T}(s) Q p(s) d s, \int_{0}^{t_{r}} p^{T}(s) \mathcal{S} u(s) d s$ and $\int_{0}^{t_{r}} u^{T}(s) \mathcal{R} u(s) d s$, respectively. 
The connection (26) can be defined by the following dissipativity performance

$$
\begin{aligned}
& \mathbb{J}_{\gamma, t_{r}}= \\
& \int_{0}^{t_{r}} \mathbb{E}\left\{\left[\left(\begin{array}{l}
p(s) \\
u(s)
\end{array}\right)^{T}\left(\begin{array}{cc}
Q & S \\
\mathcal{L}^{2} & \mathcal{R}-\gamma \mathbf{I}
\end{array}\right)\left(\begin{array}{l}
p(s) \\
u(s)
\end{array}\right)\right\} d s .\right.
\end{aligned}
$$

Lemma 2.3. For a positive-definite matrix $\mathbf{O}>0$ and a differentiable function $\left\{q(s) \mid s \in\left[\vartheta_{1}, \vartheta_{2}\right]\right\}$, the following integral inequality holds

$$
\begin{aligned}
& \int_{\vartheta_{1}}^{\vartheta_{2}} \dot{q}^{T}(s) \mathbf{O} \dot{q}(s) d s \geq \frac{1}{\vartheta_{2}-\vartheta_{1}} \mathscr{O}_{1}^{T} \mathbf{O} \mathscr{O}_{1}+ \\
& \frac{3}{\vartheta_{2}-\vartheta_{1}} \mathscr{O}_{2}^{T} \mathbf{O} \mathscr{O}_{2}+\frac{5}{\vartheta_{2}-\vartheta_{1}} \mathscr{O}_{3}^{T} \mathbf{O} \mathscr{O}_{3}
\end{aligned}
$$

where $\mathscr{O}_{1}=q\left(\vartheta_{2}\right)-q\left(\vartheta_{1}\right), \quad \mathscr{O}_{2}=q\left(\vartheta_{2}\right)+$ $q\left(\vartheta_{1}\right)-\frac{2}{\vartheta_{2}-\vartheta_{1}} \int_{\vartheta_{1}}^{\vartheta_{2}} q(s) d s, \mathscr{O}_{3}=q\left(\vartheta_{2}\right)-q\left(\vartheta_{1}\right)+$ $\frac{6}{\vartheta_{2}-\vartheta_{1}} \int_{\vartheta_{1}}^{\vartheta_{2}} q(s) d s-\frac{12}{\left(\vartheta_{2}-\vartheta_{1}\right)^{2}} \int_{\vartheta_{1}}^{\vartheta_{2}} \int_{u}^{\vartheta_{2}} q(s) d s d u$.

Lemma 2.4. For given positive integers $m$ and $n, a$ scalar $\alpha \in(0,1)$, a $n \times n$ - matrix $\mathbf{O}>0$, two matrices $\mathbf{Z}_{1}, \mathbf{Z}_{2} \in \mathbb{R}^{n \times m}$, and any vector $\xi \in \mathbb{R}^{m}$, define the function $\Theta(\alpha, \mathbf{O})$ described by

$$
\Theta(\alpha, \mathbf{O})=\frac{1}{\alpha} \xi^{T} \mathbf{Z}_{1}^{T} \mathbf{O} \mathbf{Z}_{1} \xi+\frac{1}{1-\alpha} \xi^{T} \mathbf{Z}_{2}^{T} \mathbf{O} \mathbf{Z}_{2} \xi .
$$

If there exists a matrix $\mathbf{G} \in \mathbb{R}^{n \times n}$ satisfying

$$
\begin{aligned}
& \left(\begin{array}{ll}
\mathbf{O} & \mathbf{G} \\
\mathbf{A} & \mathbf{O}
\end{array}\right)>0, \text { then } \\
& \quad \min _{\alpha \in(0,1)} \Theta(\alpha, \mathbf{O}) \geq\left(\begin{array}{l}
\mathbf{Z}_{1} \xi \\
\mathbf{Z}_{2} \xi
\end{array}\right)^{T}\left(\begin{array}{ll}
\mathbf{O} & \mathbf{G} \\
\mathbf{H} & \mathbf{O}
\end{array}\right)\left(\begin{array}{l}
\mathbf{Z}_{1} \xi \\
\mathbf{Z}_{2} \xi
\end{array}\right) .
\end{aligned}
$$

\section{Main results}

In this Section, the main result is given in the following Theorem (3.1), which shows that NTSMJSNNs (7) with $u(t)=0$ is globally asymptotically stable in the mean square. Firstly, if the condition (10) does not be relaxed as (18). Before giving the main results, we present the following notations for the simplicity of presentation, such as

$$
\begin{aligned}
& \Gamma_{1}=\operatorname{diag}\left(\gamma_{1}^{-}, \gamma_{2}^{-}, \ldots, \gamma_{n}^{-}\right), \\
& \Gamma_{2}=\operatorname{diag}\left(\gamma_{1}^{+}, \gamma_{2}^{+}, \ldots, \gamma_{n}^{+}\right), \\
& \Gamma_{3}=\operatorname{diag}\left(\gamma_{1}^{-} \gamma_{1}^{+}, \gamma_{2}^{-} \gamma_{2}^{+}, \ldots, \gamma_{n}^{-} \gamma_{n}^{+}\right), \\
& \Gamma_{4}=\operatorname{diag}\left(\frac{\gamma_{1}^{-}+\gamma_{1}^{+}}{2}, \frac{\gamma_{2}^{-}+\gamma_{2}^{+}}{2}, \ldots, \frac{\gamma_{n}^{-}+\gamma_{n}^{+}}{2}\right), \\
& \xi(t)=\left[q^{T}(t), \dot{q}^{T}(t-h(t)), q^{T}(t-\eta(t)),\right. \\
& q^{T}(t-\eta), f^{T}\left(\mathbf{W}_{i} q(t)\right), f^{T}\left(\mathbf{W}_{i} q(t-\eta(t))\right), \\
& f^{T}\left(\mathbf{W}_{i} q(t-\eta)\right), \frac{1}{\eta-\eta(t)} \int_{t-\eta}^{t-\eta(t)} q^{T}(s) d s, \\
& \frac{1}{\eta(t)} \int_{t-\eta(t)}^{t} q^{T}(s) d s, \\
& \frac{1}{(\eta-\eta(t))^{2}} \int_{t-\eta}^{t-\eta(t)} \int_{u}^{t} q^{T}(s) d s d u \\
& \left.\frac{1}{(\eta(t))^{2}} \int_{t-\eta(t)}^{t} \int_{u}^{t} q^{T}(s) d s d u\right]^{T}, \\
e_{i}= & {\left[0_{n \times(i-1) n}, \mathbf{I}_{n \times n}, 0_{n \times(11-i) n}\right], i=1,2,3, \ldots, 11 . }
\end{aligned}
$$

Theorem 3.1. Consider the NN (7) with nonlinear stochastic noise $\sigma(t, q(t), q(t-\eta(t)), i)$ satisfying the condition (9). For given scalars $\eta, h$ and $\mu$, the $N N$ (7) with $u(t)=0$ is globally asymptotically stable in the mean square, if there exists matrices $\mathbf{P}_{i}(i \in S)>0,\left(\begin{array}{cc}\mathbf{Q}_{11} & \mathbf{Q}_{12} \\ & \mathbf{Q}_{22}\end{array}\right)>0,\left(\begin{array}{cc}\mathbf{R}_{11} & \mathbf{R}_{12} \\ & \mathbf{R}_{22}\end{array}\right)>$ $0, \mathbf{S}>0, \mathbf{U}>0$, positive diagonal matrices $\mathbf{M}^{j}(j=$ $1,2, \ldots, 5)$, any matrices $\mathbf{G}_{j}(j=1,2, \ldots, 9)$ and positive scalar $\lambda_{i}(i \in S)$ such that the following LMIs holds for all $(i \in S)$

$$
\begin{aligned}
\mathbf{P}_{\mathbf{i}} & \leq \lambda_{i} \mathbf{I}, \\
\Theta_{1} & =\left(\begin{array}{cccc}
\Xi-\mathscr{G}^{T} \mathbb{U} \mathscr{G} & \Delta_{1}^{T} & \Delta_{2}^{T} \\
& & -\mathbf{S} & 0 \\
& & & -\mathbf{U}
\end{array}\right)<0, \\
\mathbb{U} & =\left(\begin{array}{cccccc}
\mathbf{S} & 0 & 0 & \mathbf{G}_{1} & \mathbf{G}_{2} & \mathbf{G}_{3} \\
& 3 \mathbf{S} & 0 & \mathbf{G}_{4} & \mathbf{G}_{5} & \mathbf{G}_{6} \\
& 5 \mathbf{S} & \mathbf{G}_{7} & \mathbf{G}_{8} & \mathbf{G}_{9} \\
& \mathbf{S} & 0 & 0 \\
& & & 3 \mathbf{S} & 0 \\
& & & & 5 \mathbf{S}
\end{array}\right)>0,
\end{aligned}
$$

where $\Xi=\Xi^{T}=\Xi_{11 \times 11}$ with $\Xi_{1,1}=-\mathbf{P}_{i} \mathbf{D}_{i}-$ $\left(\mathbf{P}_{i} \mathbf{D}_{i}\right)^{T}+\sum_{j=1}^{N} \pi_{i j} \mathbf{P}_{j}+Q_{11}+\mathbf{R}_{11}+\lambda_{i} \mathbf{F}_{1 i}^{T} \mathbf{F}_{1 i}-$ $\mathbf{W}_{i}^{T} \Gamma_{3} \mathbf{M}^{1} \mathbf{W}_{i}-\mathbf{W}_{i}^{T} \Gamma_{3} \mathbf{M}^{4} \mathbf{W}_{i}, \Xi_{1,2}=\mathbf{P}_{i} \mathbf{A}_{i}, \Xi_{1,3}=$ 
$\mathbf{W}_{i}^{T} \Gamma_{3} \mathbf{M}^{4} \mathbf{W}_{i}, \quad \Xi_{1,5}=\mathbf{Q}_{12}+\mathbf{R}_{12}+\mathbf{W}_{i}^{T} \Gamma_{4} \mathbf{M}^{1}+$ $\mathbf{W}_{i}^{T} \Gamma_{4} \mathbf{M}^{4}, \quad \Xi_{1,6}=\mathbf{P}_{i}-\mathbf{W}_{i}^{T} \Gamma_{4} \mathbf{M}^{4}, \quad \Xi_{2,2}=$ $-\left(1-h_{d}\right) \mathbf{S}, \quad \Xi_{3,3}=-(1-\mu) \mathbf{Q}_{11}+\lambda_{i} \mathbf{F}_{2 i}^{T} \mathbf{F}_{2 i}-$ $\mathbf{W}_{i}^{T} \Gamma_{3} \mathbf{M}^{2} \mathbf{W}_{i}-\mathbf{W}_{i}^{T} \Gamma_{3} \mathbf{M}^{4} \mathbf{W}_{i}-\mathbf{W}_{i}^{T} \Gamma_{3} \mathbf{M}^{5} \mathbf{W}_{i}, \Xi_{3,4}=$ $\mathbf{W}_{i}^{T} \Gamma_{3} \mathbf{M}^{5} \mathbf{W}_{i}, \Xi_{3,5}=-\left(\Gamma_{4} \mathbf{M}^{4} \mathbf{W}_{i}\right)^{T}, \Xi_{3,6}=-(1-$ u) $\mathbf{Q}_{12}+\mathbf{W}_{i}^{T} \Gamma_{4} \mathbf{M}^{2}+\mathbf{W}_{i}^{T} \Gamma_{4} \mathbf{M}^{4}+\mathbf{W}_{i}^{T} \Gamma_{4} \mathbf{M}^{5}, \Xi_{3,7}=$ $-\mathbf{W}_{i}^{T} \Gamma_{4} \mathbf{M}^{5}, \quad \Xi_{4,4}=-\mathbf{R}_{11}-\mathbf{W}_{i}^{T} \Gamma_{3} \mathbf{M}^{3} \mathbf{W}_{i}-$ $\mathbf{W}_{i}^{T} \Gamma_{3} \mathbf{M}^{5} \mathbf{W}_{i}, \quad \Xi_{4,6}=-\left(\Gamma_{4} \mathbf{M}^{5} \mathbf{W}_{i}\right)^{T}, \quad \Xi_{4,7}=$ $-\mathbf{R}_{12}+\mathbf{W}_{i}^{T} \Gamma_{4} \mathbf{M}^{3}+\mathbf{W}_{i}^{T} \Gamma_{4} \mathbf{M}^{5}, \Xi_{5,5}=\mathbf{Q}_{22}+\mathbf{R}_{22}-$ $\mathbf{M}^{1}-\mathbf{M}^{4}, \Xi_{5,6}=\mathbf{M}^{4}, \Xi_{6,6}=-(1-\mu) \mathbf{Q}_{22}-\mathbf{M}^{2}-$ $\mathbf{M}^{4}-\mathbf{M}^{5}, \Xi_{6,7}=\mathbf{M}^{5}, \Xi_{7,7}=-\mathbf{R}_{22}-\mathbf{M}^{3}-\mathbf{M}^{5}$,

$\Delta_{1}=\left[\begin{array}{lllllllllll}-\mathbf{D}_{i} \mathbf{S} & \mathbf{A}_{i} \mathbf{S} & 0 & 0 & 0 & \mathbf{S} & 0 & 0 & 0 & 0 & 0\end{array}\right], \Delta_{2}=$

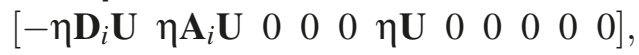

$\mathscr{G}=\left[\left(e_{3}-e_{4}\right)\left(e_{3}+e_{4}-2 e_{8}\right)\left(e_{3}-e_{4}+6 e_{8}-\right.\right.$ $\left.12 e_{10}\right)\left(e_{1}-e_{3}\right)\left(e_{1}+e_{3}-2 e_{9}\right)\left(e_{1}-e_{3}+6 e_{9}-\right.$ $\left.\left.12 e_{11}\right)\right]$.

Proof: Choose the Lyapunov function candidate for the considered system model (7) as follows

$$
\begin{aligned}
& \mathbb{V}(t, q(t), i)=\mathbb{V}_{1}(t, q(t), i)+\mathbb{V}_{2}(t, q(t), i) \\
& +\mathbb{V}_{3}(t, q(t), i)+\mathbb{V}_{4}(t, q(t), i)+\mathbb{V}_{5}(t, q(t), i)
\end{aligned}
$$

where

$$
\begin{aligned}
\mathbb{V}_{1}(t, q(t), i)= & q^{T}(t) \mathbf{P}_{i} q(t), \\
\mathbb{V}_{2}(t, q(t), i)= & \int_{t-\eta(t)}^{t}\left(\begin{array}{c}
q(s) \\
f\left(\mathbf{W}_{i} q(s)\right)
\end{array}\right)^{T} \\
& \left(\begin{array}{cc}
\mathbf{Q}_{11} & \mathbf{Q}_{12} \\
\mathbf{Q}_{22}
\end{array}\right)\left(\begin{array}{c}
q(s) \\
f\left(\mathbf{W}_{i} q(s)\right)
\end{array}\right) d s, \\
\mathbb{V}_{3}(t, q(t), i)= & \int_{t-\eta}^{t}\left(\begin{array}{c}
q(s) \\
f\left(\mathbf{W}_{i} q(s)\right)
\end{array}\right){ }^{T} \\
& \left(\begin{array}{cc}
\mathbf{R}_{11} & \mathbf{R}_{12} \\
\mathbf{R}_{22}
\end{array}\right)\left(\begin{array}{c}
q(s) \\
f\left(\mathbf{W}_{i} s q(s)\right)
\end{array}\right) d s, \\
\mathbb{V}_{4}(t, q(t), i)= & \int_{t-h(t)}^{t} \dot{q}^{T}(s) \mathbf{S} \dot{q}(s) d s, \\
\mathbb{V}_{5}(t, q(t), i)= & \eta \int_{t-\eta}^{t} \int_{u}^{t} \dot{q}^{T}(s) \mathbf{U} \dot{q}(s) d s d u . \\
\mathcal{L} \mathbb{V}(t, q(t), i)= & \mathbb{V}_{t}(t, q(t), i)+\mathbb{V}_{q}(t, q(t), i) \\
& {\left[-\mathbf{D}_{i} q(t)+f\left(\mathbf{W}_{i} q(t-\eta(t))\right)\right.} \\
& \left.+\mathbf{A}_{i} \dot{q}(t-h(t))\right]+\frac{1}{2} \operatorname{tr}\left[\sigma^{T}(t,\right. \\
& q(t), q(t-\eta(t)), i) \mathbb{V}_{q q}(t, q(t), \\
& i) \sigma(t, q(t), q(t-\eta(t)), i)] \\
& +\sum_{j=1}^{N} \pi_{i j} \mathbb{V}(t, q(t), j),
\end{aligned}
$$

where

$$
\begin{aligned}
& \mathbb{V}_{t}(t, q(t), i)=\frac{\partial \mathbb{V}(t, q(t), i)}{\partial t}, \\
& \mathbb{V}_{q}(t, q(t), i)=\left(\frac{\partial \mathbb{V}(t, q(t), i)}{\partial q_{1}}, \ldots,\right. \\
& \left.\frac{\partial \mathbb{V}(t, q(t), i)}{\partial q_{n}}\right), \\
& \mathbb{V}_{q q}(t, q(t), i)=\left(\frac{\partial^{2} \mathbb{V}(t, q(t), i)}{\partial q_{i} \partial q_{j}}\right)_{n \times n} .
\end{aligned}
$$

It follows from Ito's differential rule, we have

$$
\begin{aligned}
& d \mathbb{V}_{t}(t, q(t), i)=\mathcal{L} \mathbb{V}(t, q(t), i) d t+ \\
& \mathbb{V}_{q}[\sigma(t, q(t), q(t-\eta(t)), i)] d \omega(t),
\end{aligned}
$$

where

$$
\begin{aligned}
& \mathcal{L} \mathbb{V}(t, q(t), i)=\mathcal{L} \mathbb{V}_{1}(t, q(t), i)+\mathcal{L} \mathbb{V}_{2}(t, q(t), i) \\
& +\mathcal{L} \mathbb{V}_{3}(t, q(t), i)+\mathcal{L} \mathbb{V}_{4}(t, q(t), i)+\mathcal{L} \mathbb{V}_{5}(t, q(t), i) .
\end{aligned}
$$

Now we calculate $\mathcal{L} \mathbb{V}(t, q(t), i)$ along the trajectories of the system (7), one has

$$
\begin{aligned}
& \mathcal{L} \mathbb{V}_{1}(t, q(t), i)=2 q^{T}(t) \mathbf{P}_{i}\left[-\mathbf{D}_{i} q(t)+f\left(\mathbf{W}_{i}\right.\right. \\
& \left.q(t-\eta(t)))+\mathbf{A}_{i} \dot{q}(t-h(t))\right]+q^{T}(t) \\
& \left(\sum_{j=1}^{N} \pi_{i j} \mathbf{P}_{j}\right) q(t)+\operatorname{tr}\left(\boldsymbol{\sigma}^{T}(t, q(t),\right. \\
& q(t-\eta(t)), i) \mathbf{P}_{i}(\sigma(t, q(t), q(t-\eta(t)), i), \\
& \mathcal{L} \mathbb{V}_{2}(t, q(t), i) \leq\left(\begin{array}{c}
q(t) \\
f\left(\mathbf{W}_{i} q(t)\right)
\end{array}\right)^{T} \\
& \left(\begin{array}{cc}
\mathbf{Q}_{11} & \mathbf{Q}_{12} \\
& \mathbf{Q}_{22}
\end{array}\right)\left(\begin{array}{c}
q(t) \\
f\left(\mathbf{W}_{i} q(t)\right)
\end{array}\right) \\
& -(1-\dot{\eta}(t))\left(\begin{array}{c}
q(t-\eta(t)) \\
f\left(\mathbf{W}_{i} q(t-\eta(t))\right)
\end{array}\right)^{T} \\
& \left(\begin{array}{cc}
\mathbf{Q}_{11} & \mathbf{Q}_{12} \\
& \mathbf{Q}_{22}
\end{array}\right)\left(\begin{array}{c}
q(t-\eta(t)) \\
f\left(\mathbf{W}_{i} q(t-\eta(t))\right)
\end{array}\right) \text {, } \\
& \mathcal{L} \mathbb{V}_{3}(t, q(t), i)=\left(\begin{array}{c}
q(t) \\
f\left(\mathbf{W}_{i} q(t)\right)
\end{array}\right)^{T} \\
& \left(\begin{array}{cc}
\mathbf{R}_{11} & \mathbf{R}_{12} \\
& \mathbf{R}_{22}
\end{array}\right)\left(\begin{array}{c}
q(t) \\
f\left(\mathbf{W}_{i} q(t)\right)
\end{array}\right) \\
& -\left(\begin{array}{c}
q(t-\eta) \\
f\left(\mathbf{W}_{i} q(t-\eta)\right)
\end{array}\right)^{T} \\
& \left(\begin{array}{cc}
\mathbf{R}_{11} & \mathbf{R}_{12} \\
& \mathbf{R}_{22}
\end{array}\right)\left(\begin{array}{c}
q(t-\eta) \\
f\left(\mathbf{W}_{i} q(t-\eta)\right)
\end{array}\right), \\
& \mathcal{L} \mathbb{V}_{4}(t, q(t), i) \leq \dot{q}^{T}(t) \mathbf{S} \dot{q}(t)-(1-\dot{h}(t)) \\
& \dot{q}^{T}(t-h(t)) \mathbf{S} \dot{q}(t-h(t)),
\end{aligned}
$$




$$
\begin{aligned}
& \mathcal{L} \mathbb{V}_{5}(t, q(t), i)=\eta^{2} \dot{q}^{T}(t) \mathbf{U} \dot{q}(t) \\
& -\eta \int_{t-\eta}^{t} \dot{q}^{T}(s) \mathbf{U} \dot{q}(s) d s \\
& =\eta^{2} \dot{q}^{T}(t) \mathbf{U} \dot{q}(t)-\eta \int_{t-\eta}^{t-\eta(t)} \dot{q}^{T}(s) \mathbf{U} \dot{q}(s) d s \\
& -\eta \int_{t-\eta(t)}^{t} \dot{q}^{T}(s) \mathbf{U} \dot{q}(s) d s \\
& \leq \eta^{2} \dot{q}^{T}(t) \mathbf{U} \dot{q}(t)-\frac{\eta}{\eta-\eta(t)}\left[\mathscr{C}_{1}^{T}(t) \mathbf{U} \mathscr{C}_{1}(t)+\right. \\
& \left.\mathscr{C}_{2}^{T}(t) 3 \mathbf{U} \mathscr{C}_{2}(t)+\mathscr{C}_{3}^{T}(t) 5 \mathbf{U} \mathscr{C}_{3}(t)\right]-\frac{\eta}{\eta(t)}\left[\mathscr{C}_{4}^{T}(t)\right. \\
& \left.\mathbf{U} \mathscr{C}_{4}(t)+\mathscr{C}_{5}^{T}(t) 3 \mathbf{U} \mathscr{C}_{5}(t)+\mathscr{C}_{6}^{T}(t) 5 \mathbf{U} \mathscr{C}_{6}(t)\right] \\
& =\eta^{2} \dot{q}^{T}(t) \mathbf{U} \dot{q}(t)-\frac{\eta}{\eta-\eta(t)}\left(\begin{array}{c}
\mathscr{C}_{1}(t) \\
\mathscr{C}_{2}(t) \\
\mathscr{C}_{3}(t)
\end{array}\right)^{T} \widehat{\mathbf{U}} \\
& \left(\begin{array}{l}
\mathscr{C}_{1}(t) \\
\mathscr{C}_{2}(t) \\
\mathscr{C}_{3}(t)
\end{array}\right)-\frac{\eta}{\eta(t)}\left(\begin{array}{l}
\mathscr{C}_{4}(t) \\
\mathscr{C}_{5}(t) \\
\mathscr{C}_{6}(t)
\end{array}\right)^{T} \widehat{\mathbf{U}}\left(\begin{array}{l}
\mathscr{C}_{4}(t) \\
\mathscr{C}_{5}(t) \\
\mathscr{C}_{6}(t)
\end{array}\right) \\
& =\eta^{2} \dot{q}^{T}(t) \mathbf{U} \dot{q}(t)-\left(\begin{array}{c}
\mathscr{C}_{1}(t) \\
\mathscr{C}_{2}(t) \\
\mathscr{C}_{3}(t)
\end{array}\right)^{T} \widehat{\mathbf{U}}\left(\begin{array}{c}
\mathscr{C}_{1}(t) \\
\mathscr{C}_{2}(t) \\
\mathscr{C}_{3}(t)
\end{array}\right)- \\
& \frac{\eta(t)}{\eta-\eta(t)}\left(\begin{array}{c}
\mathscr{C}_{1}(t) \\
\mathscr{C}_{2}(t) \\
\mathscr{C}_{3}(t)
\end{array}\right)^{T} \widehat{\mathbf{U}}\left(\begin{array}{c}
\mathscr{C}_{1}(t) \\
\mathscr{C}_{2}(t) \\
\mathscr{C}_{3}(t)
\end{array}\right) \\
& -\left(\begin{array}{l}
\mathscr{C}_{4}(t) \\
\mathscr{C}_{5}(t) \\
\mathscr{C}_{6}(t)
\end{array}\right)^{T} \widehat{\mathbf{U}}\left(\begin{array}{l}
\mathscr{C}_{4}(t) \\
\mathscr{C}_{5}(t) \\
\mathscr{C}_{6}(t)
\end{array}\right) \\
& -\frac{\eta-\eta(t)}{\eta(t)}\left(\begin{array}{c}
\mathscr{C}_{4}(t) \\
\mathscr{C}_{5}(t) \\
\mathscr{C}_{6}(t)
\end{array}\right)^{T} \widehat{\mathbf{U}}\left(\begin{array}{c}
\mathscr{C}_{4}(t) \\
\mathscr{C}_{5}(t) \\
\mathscr{C}_{6}(t)
\end{array}\right) .
\end{aligned}
$$$$
\text { If }\left(\begin{array}{cc}
\widehat{\mathbf{U}} & \widehat{\mathbf{G}} \\
& \widehat{\mathbf{U}}
\end{array}\right)>0 \text { the following inequality is satisfied }
$$
by Lemma (2.4)

$$
\begin{aligned}
& \left(\begin{array}{c}
\sqrt{\frac{\eta(t)}{\eta-\eta(t)}}\left(\begin{array}{l}
\mathscr{C}_{1}(t) \\
\mathscr{C}_{2}(t) \\
\mathscr{C}_{3}(t)
\end{array}\right) \\
-\sqrt{\frac{\eta-\eta(t)}{\eta(t)}}\left(\begin{array}{l}
\mathscr{C}_{4}(t) \\
\mathscr{C}_{5}(t) \\
\mathscr{C}_{6}(t)
\end{array}\right)
\end{array}\right)\left(\begin{array}{ll}
\widehat{\mathbf{U}} & \widehat{\mathbf{G}} \\
& \widehat{\mathbf{U}}
\end{array}\right) \\
& \left(\begin{array}{c}
\sqrt{\frac{\eta(t)}{\eta-\eta(t)}}\left(\begin{array}{l}
\mathscr{C}_{1}(t) \\
\mathscr{C}_{2}(t) \\
\mathscr{C}_{3}(t)
\end{array}\right) \\
-\sqrt{\frac{\eta-\eta(t)}{\eta(t)}}\left(\begin{array}{l}
\mathscr{C}_{4}(t) \\
\mathscr{C}_{5}(t) \\
\mathscr{C}_{6}(t)
\end{array}\right)
\end{array}\right) \geq 0,
\end{aligned}
$$

which implies

$$
\begin{aligned}
& -\frac{\eta(t)}{\eta-\eta(t)}\left(\begin{array}{l}
\mathscr{C}_{1}(t) \\
\mathscr{C}_{2}(t) \\
\mathscr{C}_{3}(t)
\end{array}\right)^{T} \widehat{\mathbf{U}}\left(\begin{array}{l}
\mathscr{C}_{1}(t) \\
\mathscr{C}_{2}(t) \\
\mathscr{C}_{3}(t)
\end{array}\right) \\
& -\frac{\eta-\eta(t)}{\eta(t)}\left(\begin{array}{l}
\mathscr{C}_{4}(t) \\
\mathscr{C}_{5}(t) \\
\mathscr{C}_{6}(t)
\end{array}\right)^{T} \widehat{\mathbf{U}}\left(\begin{array}{l}
\mathscr{C}_{4}(t) \\
\mathscr{C}_{5}(t) \\
\mathscr{C}_{6}(t)
\end{array}\right) \\
& \leq-\left(\begin{array}{l}
\mathscr{C}_{1}(t) \\
\mathscr{C}_{2}(t) \\
\mathscr{C}_{3}(t)
\end{array}\right)^{T} \widehat{\mathbf{G}}\left(\begin{array}{l}
\mathscr{C}_{4}(t) \\
\mathscr{C}_{5}(t) \\
\mathscr{C}_{6}(t)
\end{array}\right)- \\
& \left(\begin{array}{l}
\mathscr{C}_{4}(t) \\
\mathscr{C}_{5}(t) \\
\mathscr{C}_{6}(t)
\end{array}\right)^{T} \widehat{\mathbf{G}}^{T}\left(\begin{array}{l}
\mathscr{C}_{1}(t) \\
\mathscr{C}_{2}(t) \\
\mathscr{C}_{3}(t)
\end{array}\right) .
\end{aligned}
$$

Then, we can get from (37) and (40) that

$$
\begin{aligned}
& \mathcal{L} \mathbb{V}_{5}(t, q(t), i) \leq \eta^{2} \dot{q}^{T}(t) \mathbf{U} \dot{q}(t)- \\
& \left(\begin{array}{l}
\mathscr{C}_{1}(t) \\
\mathscr{C}_{2}(t) \\
\mathscr{C}_{3}(t)
\end{array}\right)^{T} \widehat{\mathbf{U}}\left(\begin{array}{l}
\mathscr{C}_{1}(t) \\
\mathscr{C}_{2}(t) \\
\mathscr{C}_{3}(t)
\end{array}\right)- \\
& \left(\begin{array}{l}
\mathscr{C}_{4}(t) \\
\mathscr{C}_{5}(t) \\
\mathscr{C}_{6}(t)
\end{array}\right)^{T} \widehat{\mathbf{U}}\left(\begin{array}{l}
\mathscr{C}_{4}(t) \\
\mathscr{C}_{5}(t) \\
\mathscr{C}_{6}(t)
\end{array}\right)- \\
& \left(\begin{array}{l}
\mathscr{C}_{1}(t) \\
\mathscr{C}_{2}(t) \\
\mathscr{C}_{3}(t)
\end{array}\right)^{T} \widehat{\mathbf{G}}\left(\begin{array}{l}
\mathscr{C}_{4}(t) \\
\mathscr{C}_{5}(t) \\
\mathscr{C}_{6}(t)
\end{array}\right)- \\
& \left(\begin{array}{l}
\mathscr{C}_{4}(t) \\
\mathscr{C}_{5}(t) \\
\mathscr{C}_{6}(t)
\end{array}\right)^{T} \widehat{\mathbf{G}}\left(\begin{array}{l}
\mathscr{C}_{1}(t) \\
\mathscr{C}_{2}(t) \\
\mathscr{C}_{3}(t)
\end{array}\right) \\
& =\eta^{2} \dot{q}^{T}(t) \mathbf{U} \dot{q}(t)-\mathscr{C}^{T}(t)\left(\begin{array}{ll}
\widehat{\mathbf{U}} & \widehat{\mathbf{G}} \\
\widehat{\mathbf{U}}
\end{array}\right) \mathscr{C}(t),
\end{aligned}
$$

where

$\mathscr{C}(t)=\left[\mathscr{C}_{1}^{T}(t), \mathscr{C}_{2}^{T}(t), \mathscr{C}_{3}^{T}(t), \mathscr{C}_{4}^{T}(t), \mathscr{C}_{5}(t), \mathscr{C}_{6}^{T}(t)\right]^{T}$, $\mathscr{C}_{1}(t)=[q(t-\eta(t))-q(t-\eta)], \mathscr{C}_{2}(t)=[q(t-$ $\left.\eta(t))+q(t-\eta)-\frac{2}{(\eta-\eta(t))} \int_{t-\eta}^{t-\eta(t)} q(s) d s\right], \mathscr{C}_{3}(t)=$ $\left[q(t-\eta(t))-q(t-\eta)+\frac{6}{(\eta-\eta(t))} \int_{t-\eta}^{t-\eta(t)} q(s) d s-\right.$ $\left.\frac{12}{(\eta-\eta(t))^{2}} \int_{t-\eta}^{t-\eta(t)} \int_{u}^{t} q(s) d s d u\right], \quad \mathscr{C}_{4}(t)=[q(t)-$ $q(t-\eta(t))], \quad \mathscr{C}_{5}(t)=[q(t)+q(t-\eta(t))-$ $\left.\frac{2}{\eta(t)} \int_{t-\eta(t)}^{t} q(s) d s\right], \quad \mathscr{C}_{6}(t)=[q(t)-q(t-\eta(t))+$ $\left.\frac{6}{\eta(t)} \int_{t-\eta(t)}^{t} q(s) d s-\frac{12}{\eta(t)^{2}} \int_{t-\eta(t)}^{t} \int_{u}^{t} q(s) d s d u\right], \quad \widehat{\mathbf{U}}=$ $\left(\begin{array}{ccc}\mathbf{U} & 0 & 0 \\ 0 & 3 \mathbf{U} & 0 \\ 0 & 0 & 5 \mathbf{U}\end{array}\right), \widehat{\mathbf{G}}=\left(\begin{array}{ccccc}\mathbf{G}_{1} & \mathbf{G}_{2} & \mathbf{G}_{3} & & \\ \mathbf{G}_{4} & \mathbf{G}_{5} & \mathbf{G}_{6} \mathbf{G}_{7} & \mathbf{G}_{8} & \mathbf{G}_{9}\end{array}\right)$. 
From the assumption $\mathbf{A 3}$ and (29), we can get

$\operatorname{tr}\left(\sigma^{T}(t, q(t), q(t-\eta(t)), i) \mathbf{P}_{i}(\sigma(t, q(t)\right.$,

$q(t-\eta(t)), i)$

$\leq \lambda_{i} \operatorname{tr}\left(\sigma^{T}(t, q(t), q(t-\eta(t)), i)(\sigma(t, q(t)\right.$,

$q(t-\eta(t)), i)$

$\leq \lambda_{i}\left[q^{T}(t) \mathbf{F}_{1 i}^{T} \mathbf{F}_{1 i} q(t)+q^{T}(t-\eta(t)) \mathbf{F}_{2 i}^{T}\right.$

$\left.\mathbf{F}_{2 i} q(t-\eta(t))\right]$.

Moreover, it follows from A2, we have

$$
\begin{aligned}
& {\left[f_{j}\left(\mathbf{W}_{i j} u\right)-f_{j}\left(\mathbf{W}_{i j} v\right)-\gamma_{j}^{-}\left(\mathbf{W}_{i j} u-\mathbf{W}_{i j} v\right)\right]} \\
& {\left[f_{j}\left(\mathbf{W}_{i j} u\right)-f_{j}\left(\mathbf{W}_{i j} v\right)-\gamma_{j}^{+}\left(\mathbf{W}_{i j} u-\mathbf{W}_{i j} v\right)\right] \leq 0,}
\end{aligned}
$$

for all $i \in S, j=1,2, \ldots, n$. Then, the following inequality holds

$$
\begin{aligned}
& \left(\begin{array}{c}
\mathbf{W}_{i j} q_{j}(t) \\
f_{j}\left(\mathbf{W}_{i j} q_{j}(t)\right)
\end{array}\right)^{T} \\
& \left(\begin{array}{cc}
\gamma_{j}^{-} \gamma_{j}^{+} e_{j} e_{j}^{T} & -\frac{\gamma_{j}^{-}+\gamma_{j}^{+}}{2} e_{j} e_{j}^{T} \\
-\frac{\gamma_{j}^{-}+\gamma_{j}^{+}}{2} e_{j} e_{j}^{T} & e_{j} e_{j}^{T}
\end{array}\right) \\
& \left(\begin{array}{c}
\mathbf{W}_{i j} q_{j}(t) \\
f_{j}\left(\mathbf{W}_{i j} q_{j}(t)\right)
\end{array}\right) \leq 0 .
\end{aligned}
$$

Let, for any positive diagonal matrices $\mathbf{M}^{1}=$ $\operatorname{diag}\left\{m_{1}^{1}, m_{2}^{1}, \ldots, m_{n}^{1}\right\}$, it is easy to see that

$$
\begin{aligned}
0 \leq & -\sum_{j=1}^{n} m_{j}^{1}\left(\begin{array}{c}
\mathbf{W}_{i j} q_{j}(t) \\
f_{j}\left(\mathbf{W}_{i j} q_{j}(t)\right)
\end{array}\right)^{T} \\
& \left(\begin{array}{cc}
\gamma_{j}^{-} \gamma_{j}^{+} e_{j} e_{j}^{T} & -\frac{\gamma_{j}^{-}+\gamma_{j}^{+}}{2} e_{j} e_{j}^{T} \\
-\frac{\gamma_{j}^{-}+\gamma_{j}^{+}}{2} e_{j} e_{j}^{T} & e_{j} e_{j}^{T}
\end{array}\right) \\
& \left(\begin{array}{c}
\mathbf{W}_{i j} q_{j}(t) \\
f_{j}\left(\mathbf{W}_{i j} q_{j}(t)\right)
\end{array}\right),
\end{aligned}
$$

where $e_{j}$ denote the unit column vector having 1 on its $j$ th row and zero elsewhere

For $j=1, \ldots, n$, the condition is equivalent to

$$
\begin{aligned}
0 \leq & -\left(\begin{array}{c}
\mathbf{W}_{i} q(t) \\
f\left(\mathbf{W}_{i} q(t)\right)
\end{array}\right)^{T}\left(\begin{array}{cc}
\Gamma_{3} \mathbf{M}^{1} & -\Gamma_{4} \mathbf{M}^{1} \\
& \mathbf{M}^{1}
\end{array}\right) \\
& \left(\begin{array}{c}
\mathbf{W}_{i} q(t) \\
f\left(\mathbf{W}_{i} q(t)\right)
\end{array}\right) .
\end{aligned}
$$

Similar to the above, for any positive diagonal matrices $\mathbf{M}^{j}=\operatorname{diag}\left\{m_{1}^{j}, m_{2}^{j}, \ldots, m_{n}^{j}\right\} j=2,3,4,5$, we can obtain the following inequalities:

$$
\begin{aligned}
& 0 \leq-\left(\begin{array}{c}
\mathbf{W}_{i} q(t-\eta) \\
f\left(\mathbf{W}_{i} q(t-\eta)\right)
\end{array}\right)^{T}\left(\begin{array}{cc}
\Gamma_{3} \mathbf{M}^{3} & -\Gamma_{4} \mathbf{M}^{3} \\
& \mathbf{M}^{3}
\end{array}\right) \\
& \left(\begin{array}{c}
\mathbf{W}_{i} q(t-\eta) \\
f\left(\mathbf{W}_{i} q(t-\eta)\right)
\end{array}\right),
\end{aligned}
$$

$$
\begin{aligned}
& 0 \leq-\left(\begin{array}{c}
\mathbf{W}_{i} q(t) \\
f\left(\mathbf{W}_{i} q(t)\right) \\
\mathbf{W}_{i} q(t-\eta(t)) \\
f\left(\mathbf{W}_{i} q(t-\eta(t))\right)
\end{array}\right)^{T} \\
& \left(\begin{array}{cccc}
\Gamma_{3} \mathbf{M}^{4} & -\Gamma_{4} \mathbf{M}^{4} & -\Gamma_{3} \mathbf{M}^{4} & \Gamma_{4} \mathbf{M}^{4} \\
& \mathbf{M}^{4} & \Gamma_{4} \mathbf{M}^{4} & -\mathbf{M}^{4} \\
& & \Gamma_{3} \mathbf{M}^{4} & -\Gamma_{4} \mathbf{M}^{4} \\
& & \mathbf{M}^{4}
\end{array}\right) \\
& \left(\begin{array}{c}
\mathbf{W}_{i} q(t) \\
f\left(\mathbf{W}_{i} q(t)\right) \\
\mathbf{W}_{i} q(t-\eta(t)) \\
f\left(\mathbf{W}_{i} q(t-\eta(t))\right)
\end{array}\right) \\
& 0 \leq-\left(\begin{array}{c}
\mathbf{W}_{i} q(t-\eta(t)) \\
f\left(\mathbf{W}_{i} q(t-\eta(t))\right) \\
\mathbf{W}_{i} q(t-\eta) \\
f\left(\mathbf{W}_{i} q(t-\eta)\right)
\end{array}\right)^{T} \\
& \left(\begin{array}{cccc}
\Gamma_{3} \mathbf{M}^{5} & -\Gamma_{4} \mathbf{M}^{5} & -\Gamma_{3} \mathbf{M}^{5} & \Gamma_{4} \mathbf{M}^{5} \\
& \mathbf{M}^{5} & \Gamma_{4} \mathbf{M}^{5} & -\mathbf{M}^{5} \\
& & \Gamma_{3} \mathbf{M}^{5} & -\Gamma_{4} \mathbf{M}^{5} \\
& & \mathbf{M}^{5}
\end{array}\right) \\
& \left(\begin{array}{c}
\mathbf{W}_{i} q(t-\eta(t)) \\
f\left(\mathbf{W}_{i} q(t-\eta(t))\right) \\
\mathbf{W}_{i} q(t-\eta) \\
f\left(\mathbf{W}_{i} q(t-\eta)\right)
\end{array}\right) .
\end{aligned}
$$

Hence, according to (3)-(3), one has

$$
\mathcal{L} \mathbb{V}(t, q(t), i) \leq \xi^{T}(t) \Theta_{1} \xi(t) .
$$

Then, we have

$$
\begin{aligned}
& d \mathbb{V}_{t}(t, q(t), i) \leq \xi^{T}(t) \Theta_{1} \xi(t)+ \\
& \mathbb{V}_{q}[\sigma(t, q(t), q(t-\eta(t)), i)] d \omega(t)
\end{aligned}
$$

From $\Theta_{1}<0$ in (30), there exist a scalar $\beta>0$ such that

$$
\Theta_{1}+\operatorname{diag}\{\beta \mathbf{I} 00000000000000\}<0 .
$$

Taking mathematical expectation we have

$$
\frac{d \mathbb{E} \mathbb{V}_{t}(t, q(t), i)}{d t} \leq \mathbb{E}\left[\xi^{T}(t) \Theta_{1} \xi(t)\right] \leq-\beta\|q(t)\|^{2} .
$$

Hence, the NTSMJSNNs (7) with $u(t)=0$ is globally asymptotically stable in the mean square. This ends the proof. 
Secondly, if the condition (10) can be relaxed as (18), then the following Theorem (3.2) can be obtained.

Theorem 3.2. Consider the $N N$ (7) with nonlinear stochastic noise $\sigma(t, q(t), q(t-\eta(t)), i)$ satisfying the condition (9). Assume that $\sigma(t, q(t), q(t-$ $\eta(t)), i)$, the matrices $\mathbf{F}_{1 i}$ and $\mathbf{F}_{2 i}$ satisfy (15) and (17), respectively. For given scalars $\eta, h$ and $\mu$, the $N N(7)$ with $u(t)=0$ is globally asymptotically stable in the mean square, if there exists matrices $\mathbf{P}_{i}(i \in S)>0,\left(\begin{array}{cc}\mathbf{Q}_{11} & \mathbf{Q}_{12} \\ & \mathbf{Q}_{22}\end{array}\right)>0,\left(\begin{array}{cc}\mathbf{R}_{11} & \mathbf{R}_{12} \\ & \mathbf{R}_{22}\end{array}\right)>$ $0, \mathbf{S}>0, \mathbf{U}>0$, positive diagonal matrices $\Lambda_{i}(i \in$ $S), \mathbf{M}^{j}(j=1,2, \ldots, 5)$, any matrices $\mathbf{G}_{j}(j=$ $1,2, \ldots, 9)$, such that the following LMIs holds for all $(i \in S)$

$$
\begin{aligned}
\mathbf{P}_{\mathbf{i}} & \leq \Lambda_{i}, \\
\Theta_{2} & =\left(\begin{array}{cccc}
\Xi-\mathscr{G}^{T} \mathbb{U} \mathscr{G} & \Delta_{1}^{T} & \Delta_{2}^{T} \\
& & -\mathbf{S} & 0 \\
& & & -\mathbf{U}
\end{array}\right)<0, \\
\mathbb{U} & =\left(\begin{array}{cccccc}
\mathbf{S} & 0 & 0 & \mathbf{G}_{1} & \mathbf{G}_{2} & \mathbf{G}_{3} \\
3 \mathbf{S} & 0 & \mathbf{G}_{4} & \mathbf{G}_{5} & \mathbf{G}_{6} \\
& 5 \mathbf{S} & \mathbf{G}_{7} & \mathbf{G}_{8} & \mathbf{G}_{9} \\
& \mathbf{S} & 0 & 0 \\
& & & 3 \mathbf{S} & 0 \\
& & & 5 \mathbf{S}
\end{array}\right)>0,
\end{aligned}
$$

where $\Xi=\Xi^{T}=\Xi_{11 \times 11}$ with $\Xi_{1,1}=-\mathbf{P}_{i} \mathbf{D}_{i}-$ $\left(\mathbf{P}_{i} \mathbf{D}_{i}\right)^{T}+\sum_{j=1}^{N} \pi_{i j} \mathbf{P}_{j}+Q_{11}+\mathbf{R}_{11}+\Lambda_{i} \mathbf{F}_{1 i}^{T} \mathbf{F}_{1 i}-$ $\mathbf{W}_{i}^{T} \Gamma_{3} \mathbf{M}^{1} \mathbf{W}_{i}-\mathbf{W}_{i}^{T} \Gamma_{3} \mathbf{M}^{4} \mathbf{W}_{i}, \Xi_{1,2}=\mathbf{P}_{i} \mathbf{A}_{i}, \Xi_{1,3}=$ $\mathbf{W}_{i}^{T} \Gamma_{3} \mathbf{M}^{4} \mathbf{W}_{i}, \quad \Xi_{1,5}=\mathbf{Q}_{12}+\mathbf{R}_{12}+\mathbf{W}_{i}^{T} \Gamma_{4} \mathbf{M}^{1}+$ $\mathbf{W}_{i}^{T} \Gamma_{4} \mathbf{M}^{4}, \quad \Xi_{1,6}=\mathbf{P}_{i}-\mathbf{W}_{i}^{T} \Gamma_{4} \mathbf{M}^{4}, \quad \Xi_{2,2}=$ $-\left(1-h_{d}\right) \mathbf{S}, \quad \Xi_{3,3}=-(1-\mu) \mathbf{Q}_{11}+\Lambda_{i} \mathbf{F}_{2 i}^{T} \mathbf{F}_{2 i}-$ $\mathbf{W}_{i}^{T} \Gamma_{3} \mathbf{M}^{2} \mathbf{W}_{i}-\mathbf{W}_{i}^{T} \Gamma_{3} \mathbf{M}^{4} \mathbf{W}_{i}-\mathbf{W}_{i}^{T} \Gamma_{3} \mathbf{M}^{5} \mathbf{W}_{i}, \Xi_{3,4}=$ $\mathbf{W}_{i}^{T} \Gamma_{3} \mathbf{M}^{5} \mathbf{W}_{i}, \Xi_{3,5}=-\left(\Gamma_{4} \mathbf{M}^{4} \mathbf{W}_{i}\right)^{T}, \Xi_{3,6}=-(1-$  $-\mathbf{W}_{i}^{T} \Gamma_{4} \mathbf{M}^{5}, \quad \Xi_{4,4}=-\mathbf{R}_{11}-\mathbf{W}_{i}^{T} \Gamma_{3} \mathbf{M}^{3} \mathbf{W}_{i}-$ $\mathbf{W}_{i}^{T} \Gamma_{3} \mathbf{M}^{5} \mathbf{W}_{i}, \quad \Xi_{4,6}=-\left(\Gamma_{4} \mathbf{M}^{5} \mathbf{W}_{i}\right)^{T}, \quad \Xi_{4,7}=$ $-\mathbf{R}_{12}+\mathbf{W}_{i}^{T} \Gamma_{4} \mathbf{M}^{3}+\mathbf{W}_{i}^{T} \Gamma_{4} \mathbf{M}^{5}, \Xi_{5,5}=\mathbf{Q}_{22}+\mathbf{R}_{22}-$ $\mathbf{M}^{1}-\mathbf{M}^{4}, \Xi_{5,6}=\mathbf{M}^{4}, \Xi_{6,6}=-(1-\mu) \mathbf{Q}_{22}-\mathbf{M}^{2}-$ $\mathbf{M}^{4}-\mathbf{M}^{5}, \Xi_{6,7}=\mathbf{M}^{5}, \Xi_{7,7}=-\mathbf{R}_{22}-\mathbf{M}^{3}-\mathbf{M}^{5}$,

$\Delta_{1}=\left[\begin{array}{lllllllllll}-\mathbf{D}_{i} \mathbf{S} & \mathbf{A}_{i} \mathbf{S} & 0 & 0 & 0 & \mathbf{S} & 0 & 0 & 0 & 0 & 0\end{array}\right], \Delta_{2}=$

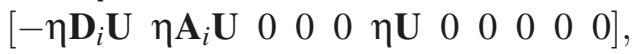

$\mathscr{G}=\left[\left(e_{3}-e_{4}\right)\left(e_{3}+e_{4}-2 e_{8}\right)\left(e_{3}-e_{4}+6 e_{8}-\right.\right.$ $\left.12 e_{10}\right)\left(e_{1}-e_{3}\right)\left(e_{1}+e_{3}-2 e_{9}\right)\left(e_{1}-e_{3}+6 e_{9}-\right.$ $\left.\left.12 e_{11}\right)\right]$.

Remark 3.3. If there is no stochastic effects and external disturbance, the system (7) can be reduced to

$$
\begin{aligned}
& \frac{d q(t)}{d t}=-\mathbf{D}_{i} q(t)+f\left(\mathbf{W}_{i} q(t-\eta(t))\right) \\
& +\mathbf{A}_{i} \dot{q}(t-h(t)) .
\end{aligned}
$$

Based on the Theorem (3.1), the following Corollary (3.3) can be obtained.

Corollary 3.4. For given scalars $\eta, h$ and $\mu$, the NN (66) is globally asymptotically stable in the mean square, if there exists matrices $\mathbf{P}_{i}(i \in S)>$ $0,\left(\begin{array}{cc}\mathbf{Q}_{11} & \mathbf{Q}_{12} \\ & \mathbf{Q}_{22}\end{array}\right)>0,\left(\begin{array}{cc}\mathbf{R}_{11} & \mathbf{R}_{12} \\ & \mathbf{R}_{22}\end{array}\right)>0, \mathbf{S}>0, \mathbf{U}>$ 0 , positive diagonal matrices $\mathbf{M}^{j}(j=1,2, \ldots, 5)$, any matrices $\mathbf{G}_{j}(j=1,2, \ldots, 9)$, such that the following LMIs holds for all $(i \in S)$;

$$
\begin{aligned}
\Theta_{3} & =\left(\begin{array}{cccc}
\Xi-\mathscr{G}^{T} \mathbb{U} \mathscr{G} & \Delta_{1}^{T} & \Delta_{2}^{T} \\
& & -\mathbf{S} & 0 \\
& & -\mathbf{U}
\end{array}\right)<0, \\
\mathbb{U} & =\left(\begin{array}{cccccc}
\mathbf{U} & 0 & 0 & \mathbf{G}_{1} & \mathbf{G}_{2} & \mathbf{G}_{3} \\
3 \mathbf{U} & 0 & \mathbf{G}_{4} & \mathbf{G}_{5} & \mathbf{G}_{6} \\
5 & \mathbf{U} & \mathbf{G}_{7} & \mathbf{G}_{8} & \mathbf{G}_{9} \\
\mathbf{U} & 0 & 0 \\
& & - & 3 & 0 \\
& & & & 5
\end{array}\right)>0,
\end{aligned}
$$

where $\Xi=\Xi^{T}=\Xi_{11 \times 11}$ with $\Xi_{1,1}=$ $-\mathbf{P}_{i} \mathbf{D}_{i}-\left(\mathbf{P}_{i} \mathbf{D}_{i}\right)^{T}+\sum_{j=1}^{N} \pi_{i j} \mathbf{P}_{j}+Q_{11}+\mathbf{R}_{11}-$ $\mathbf{W}_{i}^{T} \Gamma_{3} \mathbf{M}^{1} \mathbf{W}_{i}-\mathbf{W}_{i}^{T} \Gamma_{3} \mathbf{M}^{4} \mathbf{W}_{i}, \Xi_{1,2}=\mathbf{P}_{i} \mathbf{A}_{i}, \Xi_{1,3}=$ $\mathbf{W}_{i}^{T} \Gamma_{3} \mathbf{M}^{4} \mathbf{W}_{i}, \quad \Xi_{1,5}=\mathbf{Q}_{12}+\mathbf{R}_{12}+\mathbf{W}_{i}^{T} \Gamma_{4} \mathbf{M}^{1}+$ $\mathbf{W}_{i}^{T} \Gamma_{4} \mathbf{M}^{4}, \Xi_{1,6}=\mathbf{P}_{i}-\mathbf{W}_{i}^{T} \Gamma_{4} \mathbf{M}^{4}, \Xi_{2,2}=-(1-$ $\left.h_{d}\right) \mathbf{S}, \quad \Xi_{3,3}=-(1-\mu) \mathbf{Q}_{11}-\mathbf{W}_{i}^{T} \Gamma_{3} \mathbf{M}^{2} \mathbf{W}_{i}-$ $\mathbf{W}_{i}^{T} \Gamma_{3} \mathbf{M}^{4} \mathbf{W}_{i}-\mathbf{W}_{i}^{T} \Gamma_{3} \mathbf{M}^{5} \mathbf{W}_{i}, \Xi_{3,4}=\mathbf{W}_{i}^{T} \Gamma_{3} \mathbf{M}^{5} \mathbf{W}_{i}, \Xi_{3,5}=$ $-\left(\Gamma_{4} \mathbf{M}^{4} \mathbf{W}_{i}\right)^{T}, \Xi_{3,6}=-(1-\mu) \mathbf{Q}_{12}+\mathbf{W}_{i}^{T} \Gamma_{4} \mathbf{M}^{2}+$ $\mathbf{W}_{i}^{T} \Gamma_{4} \mathbf{M}^{4}+\mathbf{W}_{i}^{T} \Gamma_{4} \mathbf{M}^{5}, \Xi_{3,7}=-\mathbf{W}_{i}^{T} \Gamma_{4} \mathbf{M}^{5}, \Xi_{4,4}=$ $-\mathbf{R}_{11}-\mathbf{W}_{i}^{T} \Gamma_{3} \mathbf{M}^{3} \mathbf{W}_{i}-\mathbf{W}_{i}^{T} \Gamma_{3} \mathbf{M}^{5} \mathbf{W}_{i}, \quad \Xi_{4,6}=$ $-\left(\Gamma_{4} \mathbf{M}^{5} \mathbf{W}_{i}\right)^{T}, \quad \Xi_{4,7}=-\mathbf{R}_{12}+\mathbf{W}_{i}^{T} \Gamma_{4} \mathbf{M}^{3}+$ $\mathbf{W}_{i}^{T} \Gamma_{4} \mathbf{M}^{5}, \Xi_{5,5}=\mathbf{Q}_{22}+\mathbf{R}_{22}-\mathbf{M}^{1}-\mathbf{M}^{4}, \Xi_{5,6}=$ $\mathbf{M}^{4}, \Xi_{6,6}=-(1-\mu) \mathbf{Q}_{22}-\mathbf{M}^{2}-\mathbf{M}^{4}-\mathbf{M}^{5}, \Xi_{6,7}=$ $\mathbf{M}^{5}, \Xi_{7,7}=-\mathbf{R}_{22}-\mathbf{M}^{3}-\mathbf{M}^{5}$,

$\Delta_{1}=\left[\begin{array}{lllllllllll}-\mathbf{D}_{i} \mathbf{S} & \mathbf{A}_{i} \mathbf{S} & 0 & 0 & 0 & \mathbf{S} & 0 & 0 & 0 & 0 & 0\end{array}\right], \Delta_{2}=$

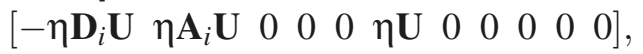

$\mathscr{G}=\left[\left(e_{3}-e_{4}\right)\left(e_{3}+e_{4}-2 e_{8}\right)\left(e_{3}-e_{4}+6 e_{8}-\right.\right.$ $\left.12 e_{10}\right)\left(e_{1}-e_{3}\right)\left(e_{1}+e_{3}-2 e_{9}\right)\left(e_{1}-e_{3}+6 e_{9}-\right.$ $\left.\left.12 e_{11}\right)\right]$.

Remark 3.5. Assume that the stochastic effects, neutral delay and external disturbance are not ap- 
pear in (7), then the system (7), becomes

$$
\frac{d q(t)}{d t}=-\mathbf{D}_{i} q(t)+f\left(\mathbf{W}_{i} q(t-\eta(t))\right) .
$$

The following Corollary (3.4) can be derived by setting $\mathbf{S}=0$ in the similar proof of Corollary (3.3).

Corollary 3.6. For given scalars $\eta$ and $\mu$, the NN (69) is globally asymptotically stable in the mean square, if there exists matrices $\mathbf{P}_{i}(i \in S)>$ $0,\left(\begin{array}{cc}\mathbf{Q}_{11} & \mathbf{Q}_{12} \\ \mathbf{*} & \mathbf{Q}_{22}\end{array}\right)>0,\left(\begin{array}{cc}\mathbf{R}_{11} & \mathbf{R}_{12} \\ & \mathbf{R}_{22}\end{array}\right)>0, \mathbf{U}>0$, positive diagonal matrices $\mathbf{M}^{j}(j=1,2, \ldots, 5)$, any matrices $\mathbf{G}_{j}(i=1,2, \ldots, 9)$, such that the following LMIs holds for all $(i \in S)$

$$
\begin{aligned}
\Theta_{4} & =\left(\begin{array}{ccccc}
\Xi-\mathscr{G}^{T} \mathbb{U} \mathscr{G} & \Delta_{1}^{T} \\
& & -\mathbf{U}
\end{array}\right)<0, \\
\mathbb{U} & =\left(\begin{array}{cccccc}
\mathbf{U} & 0 & 0 & \mathbf{G}_{1} & \mathbf{G}_{2} & \mathbf{G}_{3} \\
3 & 0 & \mathbf{G}_{4} & \mathbf{G}_{5} & \mathbf{G}_{6} \\
& 5 \mathbf{U} & \mathbf{G}_{7} & \mathbf{G}_{8} & \mathbf{G}_{9} \\
& \mathbf{U} & 0 & 0 \\
& & & 3 & 0 \\
& & & 5 &
\end{array}\right)>0,
\end{aligned}
$$

where $\Xi=\Xi^{T}=\Xi_{10 \times 10}$ with $\Xi_{1,1}=-\mathbf{P}_{i} \mathbf{D}_{i}-$ $\left(\mathbf{P}_{i} \mathbf{D}_{i}\right)^{T}+\sum_{j=1}^{N} \pi_{i j} \mathbf{P}_{j}+Q_{11}+\mathbf{R}_{11}-\mathbf{W}_{i}^{T} \Gamma_{3} \mathbf{M}^{1} \mathbf{W}_{i}-$ $\mathbf{W}_{i}^{T} \Gamma_{3} \mathbf{M}^{4} \mathbf{W}_{i}, \quad \Xi_{1,2}=\mathbf{W}_{i}^{T} \Gamma_{3} \mathbf{M}^{4} \mathbf{W}_{i}, \quad \Xi_{1,4}=$ $\mathbf{Q}_{12}+\mathbf{R}_{12}+\mathbf{W}_{i}^{T} \Gamma_{4} \mathbf{M}^{1}+\mathbf{W}_{i}^{T} \Gamma_{4} \mathbf{M}^{4}, \Xi_{1,5}=\mathbf{P}_{i}-$ $\mathbf{W}_{i}^{T} \Gamma_{4} \mathbf{M}^{4}, \Xi_{2,2}=-(1-\mu) \mathbf{Q}_{11}-\mathbf{W}_{i}^{T} \Gamma_{3} \mathbf{M}^{2} \mathbf{W}_{i}-$ $\mathbf{W}_{i}^{T} \Gamma_{3} \mathbf{M}^{4} \mathbf{W}_{i}-\mathbf{W}_{i}^{T} \Gamma_{3} \mathbf{M}^{5} \mathbf{W}_{i}, \Xi_{2,3}=\mathbf{W}_{i}^{T} \Gamma_{3} \mathbf{M}^{5} \mathbf{W}_{i}$, $\Xi_{2,4}=-\left(\Gamma_{4} \mathbf{M}^{4} \mathbf{W}_{i}\right)^{T}, \quad \Xi_{2,5}=-(1-\mu) \mathbf{Q}_{12}+$ $\mathbf{W}_{i}^{T} \Gamma_{4} \mathbf{M}^{2}+\mathbf{W}_{i}^{T} \Gamma_{4} \mathbf{M}^{4}+\mathbf{W}_{i}^{T} \Gamma_{4} \mathbf{M}^{5}, \quad \Xi_{2,6}=$ $-\mathbf{W}_{i}^{T} \Gamma_{4} \mathbf{M}^{5}, \quad \Xi_{3,3}=-\mathbf{R}_{11}-\mathbf{W}_{i}^{T} \Gamma_{3} \mathbf{M}^{3} \mathbf{W}_{i}-$ $\mathbf{W}_{i}^{T} \Gamma_{3} \mathbf{M}^{5} \mathbf{W}_{i}, \quad \Xi_{3,5}=-\left(\Gamma_{4} \mathbf{M}^{5} \mathbf{W}_{i}\right)^{T}, \quad \Xi_{3,6}=$ $-\mathbf{R}_{12}+\mathbf{W}_{i}^{T} \Gamma_{4} \mathbf{M}^{3}+\mathbf{W}_{i}^{T} \Gamma_{4} \mathbf{M}^{5}, \Xi_{4,4}=\mathbf{Q}_{22}+\mathbf{R}_{22}-$ $\mathbf{M}^{1}-\mathbf{M}^{4}, \quad \Xi_{4,5}=\mathbf{M}^{4}, \quad \Xi_{5,5}=-(1-\mu) \mathbf{Q}_{22}-$ $\mathbf{M}^{2}-\mathbf{M}^{4}-\mathbf{M}^{5}, \Xi_{5,6}=\mathbf{M}^{5}, \Xi_{6,6}=-\mathbf{R}_{22}-\mathbf{M}^{3}-$ $\mathbf{M}^{5}, \Delta_{1}=\left[\begin{array}{lllllllllll}-\eta \mathbf{D}_{i} \mathbf{U} & 0 & 0 & 0 & \eta \mathbf{U} & 0 & 0 & 0 & 0 & 0\end{array}\right]$, $\mathscr{G}=\left[\left(e_{2}-e_{3}\right)\left(e_{2}+e_{3}-2 e_{7}\right)\left(e_{2}-e_{3}+6 e_{7}-\right.\right.$ $\left.12 e_{9}\right)\left(e_{1}-e_{2}\right)\left(e_{1}+e_{2}-2 e_{8}\right)\left(e_{1}-e_{2}+6 e_{8}-\right.$ $\left.\left.12 e_{10}\right)\right]$.

\section{Dissipativity analysis}

Based on the Theorem (3.1), this Section investigate the dissipativity analysis for NTSMJSNNs (7) in the following Theorem (4.1).
Theorem 4.1. Consider the $N N$ (7) with nonlinear stochastic noise $\sigma(t, q(t), q(t-\eta(t)), i)$ satisfying the condition (9). Assume that $\sigma(t, q(t), q(t-$ $\eta(t)), i)$, the matrices $\mathbf{F}_{1 i}$ and $\mathbf{F}_{2 i}$ satisfy (15) and (17), respectively. For given scalars $\eta, h$ and $\mu$, the $N N$ (7) is $(Q, \mathcal{S}, \mathcal{R})-\gamma$ - dissipative, if there exists matrices $\mathbf{P}_{i}(i \in S)>0,\left(\begin{array}{cc}\mathbf{Q}_{11} & \mathbf{Q}_{12} \\ & \mathbf{Q}_{22}\end{array}\right)>$ $0,\left(\begin{array}{cc}\mathbf{R}_{11} & \mathbf{R}_{12} \\ & \mathbf{R}_{22}\end{array}\right)>0, \mathbf{S}>0, \mathbf{U}>0$, positive diagonal matrices $\Lambda_{i}(i \in S), \mathbf{M}^{j}(j=1,2, \ldots, 5)$, any matrices $\mathbf{G}_{j}(j=1,2, \ldots, 9)$, and scalar $\gamma>0$ such that the following LMIs holds for all $(i \in S)$

$$
\mathbf{P}_{\mathbf{i}} \leq \Lambda_{i},
$$

$$
\begin{aligned}
& \Theta_{5}=\left(\begin{array}{ccc}
\Xi-\mathscr{G}^{T} \mathbb{U} \mathscr{G} & \Delta_{1}^{T} & \Delta_{2}^{T} \\
& -\mathbf{S} & 0 \\
& & -\mathbf{U}
\end{array}\right)<0, \\
& \mathbb{U}=\left(\begin{array}{cccccc}
\mathbf{U} & 0 & 0 & \mathbf{G}_{1} & \mathbf{G}_{2} & \mathbf{G}_{3} \\
3 \mathbf{U} & 0 & \mathbf{G}_{4} & \mathbf{G}_{5} & \mathbf{G}_{6} \\
5 & \mathbf{U} & \mathbf{G}_{7} & \mathbf{G}_{8} & \mathbf{G}_{9} \\
\mathbf{U} & 0 & 0 \\
& & 3 & 0 \\
& & & 5
\end{array}\right)>0,
\end{aligned}
$$

where $\Xi=\Xi^{T}=\Xi_{12 \times 12}$ with $\Xi_{1,1}=-\mathbf{P}_{i} \mathbf{D}_{i}-$ $\left(\mathbf{P}_{i} \mathbf{D}_{i}\right)^{T}+\sum_{j=1}^{N} \pi_{i j} \mathbf{P}_{j}+Q_{11}+\mathbf{R}_{11}+\Lambda_{i} \mathbf{F}_{1 i}^{T} \mathbf{F}_{1 i}-$ $\mathbf{W}_{i}^{T} \Gamma_{3} \mathbf{M}^{1} \mathbf{W}_{i}-\mathbf{W}_{i}^{T} \Gamma_{3} \mathbf{M}^{4} \mathbf{W}_{i}, \Xi_{1,2}=\mathbf{P}_{i} \mathbf{A}_{i}, \Xi_{1,3}=$ $\mathbf{W}_{i}^{T} \Gamma_{3} \mathbf{M}^{4} \mathbf{W}_{i}, \quad \Xi_{1,5}=\mathbf{Q}_{12}+\mathbf{R}_{12}+\mathbf{W}_{i}^{T} \Gamma_{4} \mathbf{M}^{1}+$ $\mathbf{W}_{i}^{T} \Gamma_{4} \mathbf{M}^{4}, \quad \Xi_{1,6}=\mathbf{P}_{i}-\mathbf{W}_{i}^{T} \Gamma_{4} \mathbf{M}^{4}, \quad \Xi_{1,12}=$ $\mathbf{P}_{i}, \quad \Xi_{2,2}=-\left(1-h_{d}\right) \mathbf{S}, \quad \Xi_{3,3}=-(1-$ н) $\mathbf{Q}_{11}+\Lambda_{i} \mathbf{F}_{2 i}^{T} \mathbf{F}_{2 i}-\mathbf{W}_{i}^{T} \Gamma_{3} \mathbf{M}^{2} \mathbf{W}_{i}-\mathbf{W}_{i}^{T} \Gamma_{3} \mathbf{M}^{4} \mathbf{W}_{i}-$ $\mathbf{W}_{i}^{T} \Gamma_{3} \mathbf{M}^{5} \mathbf{W}_{i}, \quad \Xi_{3,4}=\mathbf{W}_{i}^{T} \Gamma_{3} \mathbf{M}^{5} \mathbf{W}_{i}, \quad \Xi_{3,5}=$ $-\left(\Gamma_{4} \mathbf{M}^{4} \mathbf{W}_{i}\right)^{T}, \Xi_{3,6}=-(1-\mu) \mathbf{Q}_{12}+\mathbf{W}_{i}^{T} \Gamma_{4} \mathbf{M}^{2}+$ $\mathbf{W}_{i}^{T} \Gamma_{4} \mathbf{M}^{4}+\mathbf{W}_{i}^{T} \Gamma_{4} \mathbf{M}^{5}, \Xi_{3,7}=-\mathbf{W}_{i}^{T} \Gamma_{4} \mathbf{M}^{5}, \Xi_{4,4}=$ $-\mathbf{R}_{11}-\mathbf{W}_{i}^{T} \Gamma_{3} \mathbf{M}^{3} \mathbf{W}_{i}-\mathbf{W}_{i}^{T} \Gamma_{3} \mathbf{M}^{5} \mathbf{W}_{i}, \quad \Xi_{4,6}=$ $-\left(\Gamma_{4} \mathbf{M}^{5} \mathbf{W}_{i}\right)^{T}, \quad \Xi_{4,7}=-\mathbf{R}_{12}+\mathbf{W}_{i}^{T} \Gamma_{4} \mathbf{M}^{3}+$ $\mathbf{W}_{i}^{T} \Gamma_{4} \mathbf{M}^{5}, \quad \Xi_{5,5}=\mathbf{Q}_{22}+\mathbf{R}_{22}-\mathbf{M}^{1}-\mathbf{M}^{4}-$ Q, $\Xi_{5,6}=\mathbf{M}^{4}, \quad \Xi_{5,12}=-\mathcal{S}, \quad \Xi_{6,6}=-(1-$  $-\mathbf{R}_{22}-\mathbf{M}^{3}-\mathbf{M}^{5}, \quad \Xi_{12,12}=-\mathcal{R}+\gamma \mathbf{I}, \quad \Delta_{1}=$ $\left[\begin{array}{llllllllllll}-\mathbf{D}_{i} \mathbf{S} & \mathbf{A}_{i} \mathbf{S} & 0 & 0 & 0 & \mathbf{S} & 0 & 0 & 0 & 0 & 0 & \mathbf{S}\end{array}\right], \Delta_{2}=$

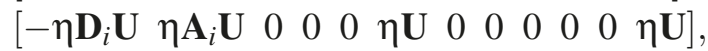

$\mathscr{G}=\left[\left(e_{3}-e_{4}\right)\left(e_{3}+e_{4}-2 e_{8}\right)\left(e_{3}-e_{4}+6 e_{8}-\right.\right.$ $\left.12 e_{10}\right)\left(e_{1}-e_{3}\right)\left(e_{1}+e_{3}-2 e_{9}\right)\left(e_{1}-e_{3}+6 e_{9}-\right.$ $\left.\left.12 e_{11}\right)\right]$.

Proof: To derive the dissipativity investigation, we take the comparable LKFs (33) and define the fol- 
lowing dissipativity condition

$$
\begin{aligned}
& \mathbb{J}_{\gamma, t_{r}}=\int_{0}^{t_{r}} \mathbb{E}\left\{\left[\left(\begin{array}{l}
p(t) \\
u(t)
\end{array}\right)^{T}\left(\begin{array}{cc}
\mathcal{Q} & \mathcal{S} \\
\star & \mathcal{R}-\gamma I
\end{array}\right)\right.\right. \\
& \left.\left(\begin{array}{l}
p(t) \\
u(t)
\end{array}\right)\right\} d t .
\end{aligned}
$$

Following the proof of Theorem (3.1), we get

$$
\begin{aligned}
& \int_{0}^{t_{r}} \mathbb{E}\{\mathcal{L} \mathbb{V}(t, q(t), i)\} d t-\mathbb{J}_{\gamma, t_{r}} \leq \\
& \int_{0}^{t_{r}} \mathbb{E}\left\{\widehat{\xi}^{T}(t) \Theta_{5} \widehat{\xi}(t)\right\} d t,
\end{aligned}
$$

where $\widehat{\xi}(t)=\left[\xi^{T}(t), \quad u^{T}(t)\right]^{T}$. It can be deduced from (73), that

$$
\int_{0}^{t_{r}} \mathbb{E}\{\mathcal{L} \mathbb{V}(t, q(t), i)\} d t \leq \mathbb{J}_{\gamma, t_{r}} .
$$

Under zero initial condition, we conclude that the condition (24) holds, which implies that the NTSMJSNNs (7) is strictly $(Q, S, R)-$ $\gamma$-dissipative. This completes the proof.

Remark 4.2. The objective of the delay-dependent stability criteria is to reduce the conservatism as much as possible. In order to handle this issue easily, the LKF approach is well considered to derive essential criteria, which plays a leading role in the less conservatism. Thus, there has been different kinds of LKFs have been introduced to derive the effective stability criteria [17-34]. Nevertheless, when calculating the upper bound of the derivative of LKF, some helpful terms are neglected. For illustrate, the term $\eta \int_{t-\eta}^{t} \int_{u}^{t} \dot{q}^{T}(s) \mathbf{U} \dot{q}(s) d s d u$ is ignored in $[20,36,24,25]$. However, in the previous works $[3,16,28,30,39]$ such term is taken into the account of LKF and the derivative of such LKF $\eta \int_{t-\eta}^{t} \int_{u}^{t} \dot{q}^{T}(s) \mathbf{U} \dot{q}(s) d s d u$ is often estimated as $\eta^{2} \dot{q}^{T}(t) \mathbf{U} \dot{q}(t)-\eta \int_{t-\eta}^{t} \int_{u}^{t} \dot{q}^{T}(s) \mathbf{U} \dot{q}(s) d s$, which may lead to reducing the conservatism as much as possible. Thus, there is still room for further analysis and reduce the conservatism.

Remark 4.3. In order to further reduce the conservatism, some useful delay-dependent integral term $\mathbb{V}_{5}(t, q(t), i)$ is considered into the account of LKF. Moreover, the integral terms in derivative of $\mathcal{L} \mathbb{V}_{5}(t, q(t), i)$, such as $-\eta \int_{t-\eta}^{t} \dot{q}^{T}(t) \mathbf{U} \dot{q}(t) d s$ does not simply employ, the equivalent integral terms $-\eta \int_{t-\eta(t)}^{t} \dot{q}^{T}(t) \mathbf{U} \dot{q}(t) d s$ and $-\eta \int_{t-\eta}^{t-\eta(t)} \dot{q}^{T}(t) \mathbf{U} \dot{q}(t) d s$ are well considered.
By applying tighter bound integral inequality, a new set of sufficient conditions have derived in Theorem (3.1), which may reduce the conservatism of stability criteria further. In addition, the stochastic perturbations terms are well taken into the account of Theorem (3.1).

Remark 4.4. The cited Lemma (2.1) and Lemma (2.2) are the great approach to reducing conservatism when studying the stability of delayed NNs, so it has been utilized widely in the stability of time delay systems (see in $[29,41,47,48]$ and the references therein).

Remark 4.5. The dissipativity analysis includes some previously known results, which contain the $H_{\infty}$, passivity performance in a unified framework. For instance, when we choose $Q=-\mathbf{I}, \mathcal{S}=0, \mathcal{R}-$ $\gamma \mathbf{I}=\gamma^{2} \mathbf{I}$, in (73) yields globally asymptotic stability with disturbance attenuation $\gamma$. Suppose we choose $Q=0, \mathcal{S}=\mathbf{I}, \mathcal{R}-\gamma \mathbf{I}=\gamma \mathbf{I}$, in (73) yields globally asymptotic stability with passivity criterion.

\section{Illustrative examples}

Here, three numerical examples and simulations are given to show the effectiveness of the proposed results.

Example 1. Consider the system (7) with $u(t)=0$ and the nonlinear stochastic disturbance $\sigma(t, q(t), q(t-\eta(t)), i)$ satisfying (15) and the following two modes:

Mode: 1

$$
\begin{aligned}
& \mathbf{D}_{1}=\left(\begin{array}{cc}
0.5 & 0 \\
0 & 0.5
\end{array}\right), \quad \mathbf{W}_{1}=\left(\begin{array}{ll}
1.6 & 2.9 \\
0.4 & 1.6
\end{array}\right) \\
& \mathbf{A}_{1}=\left(\begin{array}{cc}
0.2 & 0.1 \\
0.3 & 0.2
\end{array}\right),
\end{aligned}
$$

Mode:2

$$
\begin{aligned}
& \mathbf{D}_{2}=\left(\begin{array}{cc}
0.7 & 0 \\
0 & 0.8
\end{array}\right), \quad \mathbf{W}_{2}=\left(\begin{array}{cc}
1.7 & 1.9 \\
0.6 & 0.6
\end{array}\right), \\
& \mathbf{A}_{2}=\left(\begin{array}{cc}
0.1 & 0.1 \\
0.2 & 0.2
\end{array}\right) .
\end{aligned}
$$

Let $\Pi=\left(\begin{array}{cc}-2 & 2 \\ 3 & -3\end{array}\right)$. If $2 \mathrm{D} \sigma(t, q(t), q(t-\eta(t)), i)$ can be described by (20), then $\operatorname{tr}\left\{\sigma^{T}(t, q(t), q(t-\right.$ $\eta(t)), i) \sigma(t, q(t), q(t-\eta(t)), i)\}$ in (9) is bounded with $\mathbf{F}_{1 i}=\mathbf{F}_{2 i}=0.4 I$. The nonlinear activation functions are chosen as $f_{j}(\cdot)=\tanh \left(q_{j}\right)(j=$ 
1,2) with $\quad \Gamma_{1}=\left(\begin{array}{cc}0.1280 & 0 \\ 0 & 0.0195\end{array}\right), \Gamma_{2}=$ $\left(\begin{array}{cc}0.3680 & 0 \\ 0 & 0.1795\end{array}\right)$. The discrete time-varying delay is considered as $\eta(t)=1.4154+0.2 \sin t$ which satisfies $\eta=1.6154$ and $\mu=0.5$. Also, letting the neutral delay $h=0.5$. Solving LMIs in Theorem (3.1) and Theorem (3.2) respectively, the MAUBs $\eta$ is obtained with various $\mu$. The obtained results are shown in Table 1. From Table 1, it is clear that Theorem (3.2) is less conservative than Theorem (3.1) due to the relaxation of the condition (10) to (18). Under the initial conditions $x(0)=[0.4,-0.4]^{T}$ the following simulation results can be obtained. Figure 1 shows that the state trajectory of system (7) without $u(t)$ and the Markovian jumping modes is plotted in Figure 2.

Example 2. Consider the system (69), with $i=1$, where the parameters of the system are given as follows

$$
\begin{aligned}
& \mathbf{D}_{1}=\left(\begin{array}{ccc}
7.3458 & 0 & 0 \\
0 & 6.9987 & 0 \\
0 & 0 & 5.5949
\end{array}\right), \\
& \mathbf{W}_{1}=\left(\begin{array}{ccc}
13.6014 & -2.9616 & -0.6936 \\
7.4736 & 21.6810 & 3.2100 \\
0.7920 & -2.6334 & -20.1300
\end{array}\right), \\
& \Gamma_{1}=\left(\begin{array}{lll}
0 & 0 & 0 \\
0 & 0 & 0 \\
0 & 0 & 0
\end{array}\right), \\
& \Gamma_{2}=\left(\begin{array}{ccc}
0.3680 & 0 & 0 \\
0 & 0.1795 & 0 \\
0 & 0 & 0.2876
\end{array}\right) .
\end{aligned}
$$

First, we show that the advantages of the proposed results. In order to handle this issue easily, we consider the same system parameters discussed in [42, $43,44,45,4]$ to the system model (69) with one mode, by using MATLAB LMI control toolbox and solving LMIs in Corollary (3.4) are feasible. The detailed comparison of MAUBs with those results discussed by various methods are given in Table 2 . From Table 2, it is clear that Corollary (3.4) is less conservative than those results discussed in $[42,43$, $44,45,4]$.

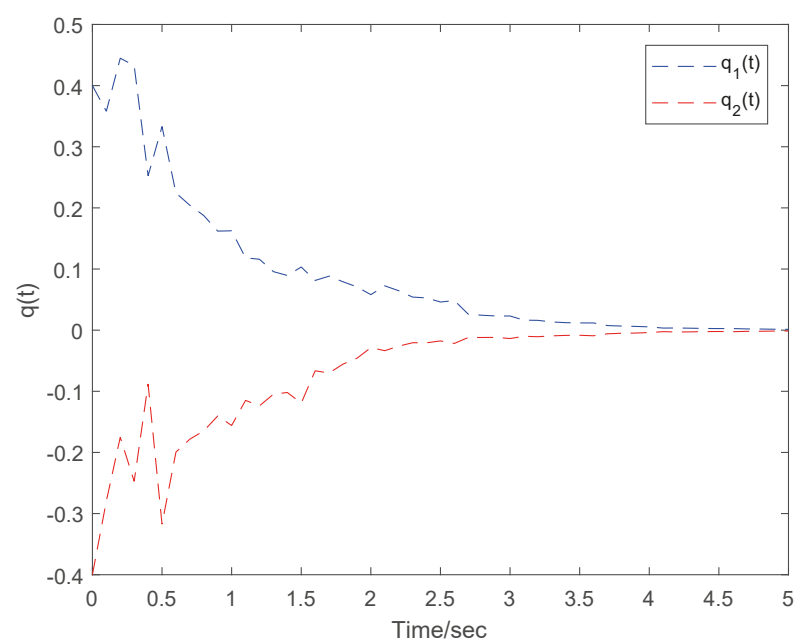

Figure 1. The state responses of system (7) with $u(t)=0$ in Example 1.

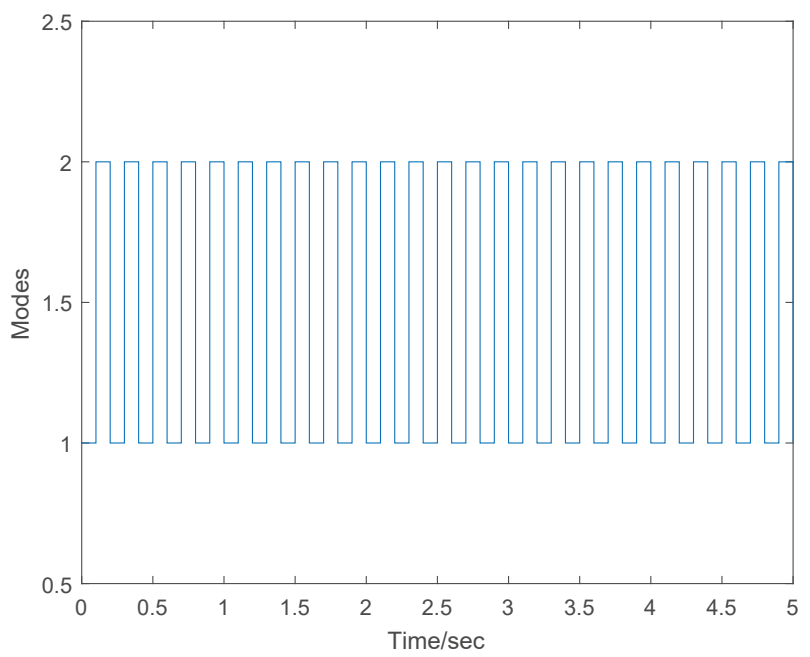

Figure 2. Markovian jumping mode of $q(t)$ in Example 1

Example 3. Consider the system (7) and the nonlinear stochastic disturbance $\sigma(t, q(t), q(t-$ $\eta(t)), i)$ satisfying (15) and the following two modes:

Mode:1

$$
\begin{aligned}
& \mathbf{D}_{1}=\left(\begin{array}{cc}
7.0214 & 0 \\
0 & 7.4367
\end{array}\right) \\
& \mathbf{W}_{1}=\left(\begin{array}{cc}
-6.4993 & -12.0275 \\
-0.6867 & 5.6614
\end{array}\right), \\
& \mathbf{A}_{1}=\left(\begin{array}{ll}
0.2 & 0.1 \\
0.3 & 0.2
\end{array}\right)
\end{aligned}
$$


Table 1. The MAUBs $\eta$ for different $\mu$

\begin{tabular}{cccccccc}
\hline Methods & $\mu$ & 0.1 & 0.3 & 0.5 & 0.7 & 0.9 & $\geq 1$ \\
\hline Theorem (3.1) & $\eta$ & 1.8643 & 1.6762 & 1.6154 & 1.3098 & 1.1003 & 1.0037 \\
Theorem (3.2) & $\eta$ & 1.8989 & 1.7014 & 1.6802 & 1.4000 & 1.1270 & 1.0716 \\
\hline
\end{tabular}

Table 2. The MAUBs $\eta$ for different $\mu$

\begin{tabular}{ccccccc}
\hline Methods & $\mu$ & 0.1 & 0.3 & 0.5 & 0.9 & $\geq 1$ \\
\hline$[42]$ & $\eta$ & 0.8402 & 0.5493 & 0.4264 & 0.3214 & - \\
{$[43]$} & $\eta$ & 0.9331 & - & 0.4268 & - & - \\
{$[44]$} & $\eta$ & 0.8411 & 0.5496 & 0.4267 & 0.3227 & - \\
{$[45]$} & $\eta$ & 1.1114 & - & 0.4514 & - & - \\
{$[4]$} & $\eta$ & 1.1153 & 0.6710 & 0.5090 & 0.4305 & - \\
Corollary (3.4) & $\eta$ & 1.8745 & 1.5750 & 1.3394 & 1.0735 & 0.9841 \\
\hline
\end{tabular}

Mode:2

$$
\begin{aligned}
& \mathbf{D}_{2}=\left(\begin{array}{cc}
6.0214 & 0 \\
0 & 6.4367
\end{array}\right), \\
& \mathbf{W}_{2}=\left(\begin{array}{cc}
-5.4993 & -11.0275 \\
-1.6867 & 4.6614
\end{array}\right), \\
& \mathbf{A}_{2}=\left(\begin{array}{cc}
0.3 & -0.1 \\
0.2 & 0.4
\end{array}\right),
\end{aligned}
$$

Moreover we take,

$$
\begin{aligned}
& \mathbf{Q}=\left(\begin{array}{ll}
1 & 0 \\
0 & 1
\end{array}\right), \mathbf{S}=\left(\begin{array}{ll}
1 & 0 \\
1 & 1
\end{array}\right), \mathbf{R}=\left(\begin{array}{ll}
3 & 0 \\
0 & 3
\end{array}\right), \\
& \Gamma_{1}=\Gamma_{2}=\left(\begin{array}{cc}
0.3680 & 0 \\
0 & 0.1795
\end{array}\right) .
\end{aligned}
$$

The purpose of this example is to show that the underlying NTSMJSNNs (7) is strictly $(Q, \mathcal{S}, \mathcal{R})-$ $\gamma$-dissipative. Let $\Pi=\left(\begin{array}{cc}-2 & 2 \\ 3 & -3\end{array}\right) . \quad$ If 2D $\sigma(t, q(t), q(t-\eta(t)), i)$ can be described by (20), then $\operatorname{tr}\left\{\sigma^{T}(t, q(t), q(t-\eta(t)), i) \sigma(t, q(t), q(t-\right.$ $\eta(t)), i)\}$ in (9) is bounded with $\mathbf{F}_{1 i}=\mathbf{F}_{2 i}=0.4 I$. Also, we choose $\eta=0.4$ and $h=0.3$, under various $\mu$, the optimal dissipativity performance $\gamma$ values is specified by Table 3, it can be obtained by Theorem (4.1).

\section{Conclusion}

In this paper, the problem of global asymptotic stability and dissipativity analysis for a class of NTSMJSNNs with time-varying delays has been investigated. In order to handle this issue, we construct an appropriate LKF with some new terms and employing integral inequality techniques, some new sufficient conditions have been obtained, which ensure that the global asymptotically stability in the mean square. Besides, the main results of this paper are further extended to the dissipativity analysis. The conditions obtained in this paper have been established in terms of strict LMIs, whose feasible solution can be verified by effective MATLAB LMI toolbox. Finally, three illustrative examples with simulation are presented to demonstrate the effectiveness of the proposed results.

\section{References}

[1] J. Liang and J. Cao, A based-on LMI stability criterion for delayed recurrent neural networks," Chaos, Solitons \& Fractals, 28 (2006) 154-160.

[2] O. M. Kwon, J. H. Park, New delay-dependent robust stability criterion for uncertain neural networks with time-varying delays, Applied Mathematics and Computation, 205 (2008) 417-427.

[3] Y. Liu, S. M. Lee, H. G. Lee, Robust delay-depent stability criteria for uncertain neural networks with two additive time-varying delay components, Neurocomputing, 151 (2015) 770-775.

[4] H. B. Zeng, J. H. Park, C. F. Zhang, W. Wang, Stability and dissipativity analysis of static neural networks with interval time-varying delay, Journal of the Franklin Institute, 352 (2015) 1284-1295.

[5] P. Muthukumar, K. Subramanian, Stability criteria for Markovian jump neural networks with mode-dependent additive time-varying delays via 
Table 3. Optimal dissipativity performance $\gamma$ for different $\mu$

\begin{tabular}{cccccccc}
\hline Method & $\mu$ & 0.1 & 0.3 & 0.5 & 0.7 & 0.9 & $\geq 1$ \\
\hline Theorem (4.1) & $\gamma$ & 1.5183 & 1.5007 & 1.4557 & 1.4010 & 1.2557 & 1.0752 \\
\hline
\end{tabular}

quadratic convex combination, Neurocomputing, 205 (2016) 75-83.

[6] T. Wang, S. Zhao, W. Zhou, W. Yu, Finite-time state estimation for delayed Hopfield neural networks with Markovian jump, Neurocomputing, 156 (2015) 193-198.

[7] X. Mao, Stochastic Differential Equations and Applications, Chichester: Horwood, 1997.

[8] Q. Zhu, J. Cao, Robust exponential stability of Markovian jump impulsive stochastic CohenGrossberg neural networks with mixed time delays, IEEE Transactions on Neural Networks, 21 (2010) 1314-1325.

[9] G. Chen, J. Xia, G. Zhuang, Delay-dependent stability and dissipativity analysis of generalized neural networks with Markovian jump parameters and two delay components, Journal of the Franklin Institute, 353 (2016) 2137-2158.

[10] Q. Zhu, J. Cao, Exponential stability of stochastic neural networks with both Markovian jump parameters and mixed time delays, IEEE Transactions on Systems, Man, and Cybernetics, Part B, 41 (2011) 341-353.

[11] Y. Chen, W. Zheng, Stability analysis of time-delay neural networks subject to stochastic perturbations, IEEE Transactions on Cybernatics, 43 (2013) 21222134.

[12] H. Tan, M. Hua, J. Chen, J. Fei, Stability analysis of stochastic Markovian switching static neural networks with asynchronous mode-dependent delays, Neurocomputing, 151 (2015) 864-872.

[13] S. Zhu, M. Shen, C. C. Lim, Robust input-to-state stability of neural networks with Markovian switching in presence of random disturbances or time delays, Neurocomputing, 249 (2017) 245-252.

[14] E. K. Boukas, Z. K. Liu, G. X. Liu, Delaydependent robust stability and $H_{\infty}$ control of jump linear systems with time-delay, International Journal of Control, 74 (2001) 329-340.

[15] Y. Y. Cao, J. Lam, L. S. Hu, Delay-dependent stochastic stability and $H_{\infty}$ analysis for time-delay systems with Markovian jumping parameters, Journal of the Franklin Institute, 340 (2003) 423-434.

[16] R. Samidurai, R. Manivannan, C. K. Ahn, H. R. Karimi, New criteria for stability of generalized neural networks including Markov jump parameters and additive time delays, IEEE Transactions on Systems, Man, and Cybernetics: Systems, 48 (2018) 485-499.

[17] S. Blythe, X. Mao, and X. Liao, Stability of stochastic delay neural networks, Journal of the Franklin Institute, 338 (2001) 481-495.

[18] Z. Zhao, Q. Song, S. He, Passivity analysis of stochastic neural networks with time-varying delays and leakage delay, Neurocomputing, 125 (2014) 2227.

[19] C. Wang, Y. Shen, Delay-dependent non-fragile robust stabilization and $H_{\infty}$ control of uncertain stochastic systems with time-varying delay and nonlinearity, Journal of the Franklin Institute, 348 (2011) 2174-2190.

[20] G. Liu, S. X. Yang, Y. Chai, W. Feng, W. Fu, Robust stability criteria for uncertain stochastic neural networks of neutral-type with interval time-varying delays, Neural Computing and Applications, 22 (2013) 349-359.

[21] R. Yang, H. Gao, P. Shi, Novel robust stability criteria for stochastic Hopfield neural networks with time delays, IEEE Transactions on Systems, Man, and Cybernetics, Part B, 39 (2009) 467-474.

[22] Q. Song, Z. Wang, Stability analysis of impulsive stochastic Cohen-Grossberg neural networks with mixed time delays, Physica A: Statistical Mechanics and its Applications, 387 (2008) 3314-3326.

[23] S. Zhu, Y. Shen, Passivity analysis of stochastic delayed neural networks with Markovian switching, Neurocomputing, 74 (2011) 1754-1761.

[24] L. Pan, J. Cao, Robust stability for uncertain stochastic neural network with delay and impulses, Neurocomputing, 94 (2012) 102-110.

[25] R. Samidurai, R. Manivannan, Delay-rangedependent passivity analysis for uncertain stochastic neural networks with discrete and distributed timevarying delays, Neurocomputing, 185 (2016) 191201.

[26] Q. Song, J. Liang, Z. Wang, Passivity analysis of discrete-time stochastic neural networks with timevarying delays, Neurocomputing, 72 (2009) 17821788.

[27] S. Zhu, Y. Shen, Robustness analysis for connection weight matrices of global exponential stability of stochastic recurrent neural networks, Neural Networks, 38 (2013) 17-22. 
[28] C. Cheng, T. Liao, J. Yan, C. Hwang, Globally asymptotic stability of a class of neutral-type neural networks with delays, IEEE Transactions on Systems, Man and CyberneticsPart B, 36 (2006) 11911195.

[29] R. Samidurai, S. Rajavel, Q. Zhu, R. Raja, H. Zhou, Robust passivity analysis for neutral-type neural networks with mixed and leakage delays, Neurocomputing, 175 (2016) 635-643.

[30] Z. Tu, J. Cao, A. Alsaedi, F. Alsaadi, Global dissipativity of memristor-based neutral type inertial neural networks, Neural Networks, 88 (2017) 125 133.

[31] R. Samidurai, S. Rajavel, R. Sriraman, J. Cao, A. Alsaedi, F. E Alsaadi, Novel results on stability analysis of neutral-type neural networks with additive time-varying delay components and leakage delay, International Journal of Control, Automation and Systems, 15 (2016) 1888-1900.

[32] R. Manivannan, R. Samidurai, J. Cao, A. Alsaedi, F. E. Alsaadi, Stability analysis of interval timevarying delayed neural networks including neutral time-delay and leakage delay, Chaos, Solitons \& Fractals, 114 (2018) 433-445.

[33] K. Mathiyalagan, R. Sakthivel and S. Marshal Anthoni, Robust exponential stability and $H_{\infty}$ control for switched neutral-type neural networks, International Journal of Adaptive Control and Signal Processing, 28 (2014) 429-443.

[34] R. Sakthivel, R. Anbuvithya, K. Mathiyalagan, A. Arunkumar and P. Prakash, New LMI-based passivity criteria for neutral-type BAM neural networks with randomly occurring uncertainties, Reports on Mathematical Physics, 72 (2013) 263-286.

[35] J. C. Willems, Dissipative dynamical systems part I: General theory, Archive for Rational Mechanics and Analysis, 45 (1972) 321-351.

[36] D. L. Hill, P. J. Moylan, Dissipative dynamical systems: basic input-output and state properties, Journal of the Franklin Institute, 309 (1980) 327-357.

[37] G. Nagamani, S. Ramasamy, Stochastic dissipativity and passivity analysis for discrete-time neural networks with probabilistic time-varying delays in the leakage term, Applied Mathematics and Computation, 289 (2016) 237-257.
[38] Z. G. Wu, Ju. H. Park, H. Su, and J. Chu, Robust dissipativity analysis of neural networks with timevarying delay and randomly occurring uncertainties, Nonlinear Dynamics, 69 (2012) 1323-1332.

[39] Z. Feng and J. Lam, Stability and dissipativity analysis of distributed delay cellular neural networks, IEEE Transactions on Neural Networks, 22 (2011) 976-981.

[40] R. Raja, U. K. Raja, R. Samidurai , and A. Leelamani, Dissipativity of discrete-time BAM stochastic neural networks with Markovian switching and impulses, Journal of the Franklin Institute, 350 (2013) 3217-3247.

[41] R. Manivannan, R. Samidurai, Q. Zhu, Further improved results on stability and dissipativity analysis of static impulsive neural networks with interval time-varying delays, Journal of the Franklin Institute, 354 (2017) 6312-6340.

[42] Z. Zuo, C. Yang, and Y. Wang, A new method for stability analysis of recurrent neural networks with interval time-varying delay, IEEE Transactions on Neural Networks, 21 (2010) 339-344.

[43] X. Li, H. Gao, and X. Yu, A unified approach to the stability of generalized static neural networks with linear fractional, IEEE Transactions Systems, Man, Cybernetics. Part B, 41 (2011) 1275-1286.

[44] Y. Q. Bai and J. Chen, New stability criteria for recurrent neural networks with interval time-varying delay, Neurocomputing, 121 (2013) 179-184.

[45] X. M. Zhang and Q. L. Han, Global asymptotic stability analysis for delayed neural networks using a matrix-based quadratic convex approach, Neural Networks, 54 (2014) 57-69.

[46] H. D. Choi, C. K. Ahn, M. T. Lim, M. K. Song, Dynamic output-feedback $H_{\infty}$ control for active halfvehicle suspension systems with time-varying input delay, International Journal of Control, Automation and Systems, 14 (2016) 59-68.

[47] P. G. Park, S. Y. Lee, W. I. Lee, Auxiliary functionbased integral inequalities for quadratic functions and their applications to time delay systems, Journal of the Franklin Institute, 352 (2015) 1378-1396.

[48] P. G. Park, J. W. Ko, C. Jeong, Reciprocally convex approach to stability of systems with time-varying delays, Automatica 47 (2011) 235-238. 


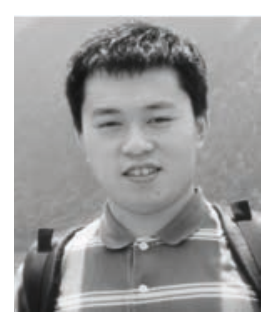

Yang Cao is currently pursuing the Ph.D. degree with the Department of Mechanical Engineering, The University of Hong Kong, Hong Kong. His current research interests include cooperative estimation and control of dynamical networks, adaptive, and optimal control. Mr. Cao received the Outstanding Undergraduate Thesis Award of Jiangsu Province, China, in 2014.

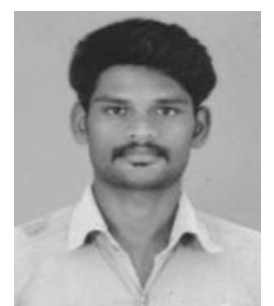

R. Sriraman is currently pursuing the Ph.D. degree with the Department of Mathematics, Thiruvalluvar University, Tamil Nadu, India. His current research interests include neural networks and time-delay systems.

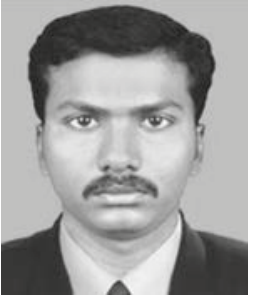

R. Samidurai received the Ph.D. degree in 2010 with the department of Mathematics, Periyar University, Salem, India. Currently, he is an Assistant Professor with the Department of Mathematics, Thiruvalluvar University, Tamil Nadu, India. He has authored over 45 research papers. He serves as a reviewer of various SCI journals. His research interests include stochastic differential equations, nonlinear systems, impulsive neural networks, networked control systems, control theory in partial differential equations and their applications. 